\title{
On the origin of Mycobacterium ulcerans, the causative agent of Buruli ulcer
}

\author{
Kenneth D Doig ${ }^{1}$, Kathryn E Holt ${ }^{1}$, Janet A M Fyfe ${ }^{3}$, Caroline J Lavender ${ }^{3}$, Miriam Eddyani ${ }^{4}$, Françoise Portaels ${ }^{4}$, \\ Dorothy Yeboah-Manu ${ }^{5}$, Gerd Pluschke ${ }^{6,7}$, Torsten Seemann ${ }^{2}$ and Timothy P Stinear ${ }^{1^{*}}$
}

\begin{abstract}
Background: Mycobacterium ulcerans is an unusual bacterial pathogen with elusive origins. While closely related to the aquatic dwelling M. marinum, M. ulcerans has evolved the ability to produce the immunosuppressive polyketide toxin mycolactone and cause the neglected tropical disease Buruli ulcer. Other mycolactone-producing mycobacteria (MPM) have been identified in fish and frogs and given distinct species designations (M. pseudoshottsii, M. shinshuense, M. liflandii and M. marinum), however the evolution of M. ulcerans and its relationship to other MPM has not been defined. Here we report the comparative analysis of whole genome sequences from 30 MPM and five M. marinum.
\end{abstract}

Results: A high-resolution phylogeny based on genome-wide single nucleotide polymorphisms (SNPs) showed that M. ulcerans and all other MPM represent a single clonal group that evolved from a common M. marinum progenitor. The emergence of the MPM was driven by the acquisition of the pMUM plasmid encoding genes for the biosynthesis of mycolactones. This change was accompanied by the loss of at least 185 genes, with a significant overrepresentation of genes associated with cell wall functions. Cell wall associated genes also showed evidence of substantial adaptive selection, suggesting cell wall remodeling has been critical for the survival of MPM. Fine-grain analysis of the MPM complex revealed at least three distinct lineages, one of which comprised a highly clonal group, responsible for Buruli ulcer in Africa and Australia. This indicates relatively recent transfer of M. ulcerans between these continents, which represent the vast majority of the global Buruli ulcer burden. Our data provide SNPs and gene sequences that can differentiate M. ulcerans lineages, suitable for use in the diagnosis and surveillance of Buruli ulcer.

Conclusions: M. ulcerans and all mycolactone-producing mycobacteria are specialized variants of a common Mycobacterium marinum progenitor that have adapted to live in restricted environments. Examination of genes lost or retained and now under selective pressure suggests these environments might be aerobic, and extracellular, where slow growth, production of an immune suppressor, cell wall remodeling, loss or modification of cell wall antigens, and biofilm-forming ability provide a survival advantage. These insights will guide our efforts to find the elusive reservoir(s) of $M$. ulcerans and to understand transmission of Buruli ulcer.

\section{Background}

The genomes of Mycobacterium ulcerans and Mycobacterium marinum are closely related, sharing $>97 \%$ overall nucleotide identity [1], but cause very different kinds of infections in humans. M. marinum causes minor skin infections, characterised by intracellular bacteria and the granulomatous lesions that are features of infection with

\footnotetext{
*Correspondence: tstinear@unimelb.edu.au

'Department of Microbiology and Immunology, University of Melbourne, Parkville, Australia

Full list of author information is available at the end of the article
}

many mycobacterial pathogens, notably Mycobacterium tuberculosis [2]. In contrast, M. ulcerans causes Buruli ulcer (BU), a slowly progressing, ulcerative disease characterized by necrosis of subcutaneous tissue. BU has a characteristic histopathology with large numbers of extracellular bacteria during the acute phase of the infection, with a marked lack of inflammatory response in advanced infection. This unusual pathology is principally mediated by an immunosuppressive polyketide called mycolactone, which is not produced by $M$. marinum or M. tuberculosis. In BU patients, mycolactone is present

\section{Biomed Central}


in cutaneous lesions but also diffuses and can be detected in serum $[3,4]$.

Two main features differentiate the genomes of $M$. ulcerans and M. marinum. The first is the pMUM megaplasmid, found in $M$. ulcerans but absent from $M$. marinum [5,6]. This plasmid harbours three large genes ( $m l s A 1: 51 \mathrm{~kb}, m l s A 2: 7.2 \mathrm{~kb}, m l s B: 43 \mathrm{~kb}$ ) that encode the polyketide synthases (PKSs) required for mycolactone synthesis [2]. The second is the insertion sequence (IS) IS2404 that is absent from M. marinum but present in high copy number $(>200)$ in $M$. ulcerans genomes. IS2404 expansion in the $M$. ulcerans genome has led to the inactivation of many genes through disruption of coding and promoter sequences and has mediated the deletion of approximately $1 \mathrm{Mbp}$ of DNA from M. ulcerans compared with $M$. marinum [1]. Together with loss of DNA, there is also evidence of extensive loss of gene function in M. ulcerans - the genome of M. ulcerans isolate Agy99 harbours 771 pseudogenes (inactivated genes), while the M. marinum genome harbours only 65 . Acquisition of foreign DNA, IS expansion, pseudogene accumulation and genome reduction are features in common with bacterial populations that have passed through an evolutionary bottleneck [7-12], suggesting there has been constriction of population size during adaptation to a new, niche environment. Analysis of the M. ulcerans Agy99 genome showed deletion or inactivation of genes expressing potent T-cell antigens, and genes required for pigment biosynthesis, anaerobiosis, and intracellular growth [1]. This profile suggested a bacterium that has adapted to a dark, extracellular environment where slow growth, loss of immunogens and production of an immunosuppressive molecule provide a selective advantage $[1,13]$. In contrast, its progenitor, $M$. marinum, has the characteristics of both a specialist bacterium that can persist within an intracellular environment as well as a generalist that can survive in extracellular conditions. A niche environment for $M$. ulcerans has not yet been demonstrated although the recent discovery that Australian native possums inhabiting BU endemic areas appear to harbour the bacteria in their gastrointestinal tracts raises some interesting possibilities [14].

The species definition of $M$. ulcerans has recently been challenged by the discovery of variously named mycobacteria that also make mycolactones but are not always associated with BU. These mycobacteria, isolated from humans, fish and frogs in diverse geographic locations (including Japan, the Mediterranean, Israel, Belgium and the United States), have been variously named Mycobacterium shinshuense, Mycobacterium marinum, Mycobacterium pseudoshottsii, and "Mycobacterium liflandii", the latter an unofficial species name with no standing in nomenclature [15-19]. Studies of these isolates using an 8- gene multi locus sequence typing (MLST) scheme and patterns of genome deletion suggest that they evolved from a $M$. marinum common ancestor and subsequently diverged into two principal lineages [20,21]. These mycolactone-producing Mycobacteria (MPM) lineages have been termed "classical" and "ancestral". The "classical" MPM lineage includes M. ulcerans isolates associated with BU from Australia, South East Asia and Africa while the "ancestral" lineage includes the fish and frog isolates as well as M. ulcerans BU isolates from Japan, China, Mexico, Surinam and French Guiana [22,23]. We recently proposed that based on their genetic coherence all MPM should be renamed M. ulcerans, a proposition we will revisit in this paper [24].

Comparative genomic analysis of closely related bacteria has facilitated dramatic improvements in our understanding of bacterial pathogen evolution [25]. Low-coverage 454 and Illumina sequencing of three $M$. ulcerans isolates from Ghana and a single isolate from Japan demonstrated the capacity of high throughput sequencing to differentiate $M$. ulcerans co-circulating in a geographic region [26]. Here we report the sequencing and analysis of whole genomes from a diversity of isolates, which are representative of what we define as the M. ulcerans-M. marinum complex (abbreviated hereafter as MuMC). That is, mycobacteria previously identified as $M$. marinum, M. ulcerans and other MPM that share $>97 \%$ nucleotide identity based on MLST [23,24]. This collection includes mycobacteria isolated from humans, possums, a bilby (small Australian mammal), fish, frogs, an insect and an armadillo from diverse geographic locations. We used whole genome comparisons to reveal the phylogenomic relationship among these mycobacteria, enabling us to investigate the hypothesis that all MPM (including $M$. ulcerans) are derived from a common ancestor. Our genome comparisons strongly supported this hypothesis and showed that the niche-adapted genomic signature of $M$. ulcerans caused by reductive evolution was established in the progenitor of all MPM before their intercontinental dispersal. We also identify DNA segments that could be used to develop molecular diagnostic tools to distinguish MPM causing BU from other members of the MuMC.

\section{Results}

Isolate selection and sequence analysis strategy

In order to capture as much diversity as possible within the MuMC and minimise phylogenetic discovery bias [27], we selected 35 mycobacteria isolates from diverse members of the MuMC for whole genome sequencing and the comparison. These isolates were recovered from a range of host organisms including humans, possums, fish, frogs, an insect and an armadillo and include 
representatives of all the major MuMC sequence types defined by MLST [21]. The majority of isolates belong to the two dominant human pathogenic clades of MPM, namely $M$. ulcerans from Africa and Australia, however MPMs that have been given various species names such as M. marinum, M. pseudoshottsii and M. liflandii were also represented. One genome was sequenced using Ion Torrent technology (single ended sequencing) and the remainder using Illumina GAIIx paired-end sequencing (Table 1 and Additional file 1: Table S1). For each isolate, genes were identified and annotated within de novo assembled contigs and then subjected to ortholog

Table 1 Isolates used in this study

\begin{tabular}{|c|c|c|c|c|c|}
\hline Identifier & Alternate identifier & Origin & Source & Year isolated & Reference \\
\hline \multicolumn{6}{|l|}{ M. ulcerans isolates } \\
\hline Mu_06-3845 & ITM001441 & Houedja, Ouinhi, Zou, Benin & Aquatic insect & 2000 & {$[28]$} \\
\hline Mu_06-3846 & ITM9711116 & Lalo, Couffo, Benin & Human & 1997 & This study \\
\hline Mu_07-1082 & ITM030216 & Adjohoun, Ouémé, Benin & Human & 2003 & This study \\
\hline Mu_1G897 & & Cayenne, French Guiana & Human & 1988 & [29] \\
\hline Mu_Agy99 & & Ga District, Ghana & Human & 1999 & [1] \\
\hline Mu_NM14.01 & & Ga District, Ghana & Human & 2001 & {$[30]$} \\
\hline Mu_NM33.04 & & Amansie West District, Ghana & Human & 2004 & {$[30]$} \\
\hline Mu_NM43.02 & & Ga District, Ghana & Human & 2002 & {$[30]$} \\
\hline Mu_NM49.02 & & Ga District, Ghana & Human & 2002 & {$[30]$} \\
\hline Mu_NM54.02 & & Ga District, Ghana & Human & 2002 & {$[30]$} \\
\hline *Mu_DL045 (M. marinum) & & Greece & Fish & 2002 & [19] \\
\hline Mu_001506 & ITM001506 & Wokon, Ouinhi, Zou, Benin & Human & 2000 & This study \\
\hline Mu_980535 & ITM980535 & Djigbé, Zé, Atlantique, Benin & Human & 1998 & This study \\
\hline Mu_000945 & ITM000945 & Hwegoudo, Zé, Atlantique, Benin & Human & 2000 & This study \\
\hline Mu_991845 & ITM991845 & Sagon, Ouinhi, Zou, Benin & Human & 1999 & This study \\
\hline *Mu_CC240299 (M. marinum) & & Israel & Fish & 1999 & [19] \\
\hline *Mu_06-3844 (M. marinum) & ITM063844 & Belgium & Fish & 2006 & [31] \\
\hline *Mu_8765 (M. shinshuense) & ITM8765 & Japan & Human & 1980 & {$[32]$} \\
\hline *Mu_JKD8071 (M. liflandii) & $128 \mathrm{FXT}$ & USA & Frog & 2004 & [33] \\
\hline${ }^{*}$ Mu_L15 (M. pseudoshottsii) & L15 & USA & Fish & 2004 & {$[17]$} \\
\hline Mu_13822-70 & & Queensland, Australia & Human & 1971 & [34] \\
\hline Mu_113 & 05152838 & Point Lonsdale, Australia & Human & 2005 & This study \\
\hline Mu_119 & 05159089 & Frankston, Australia & Human & 2005 & This study \\
\hline Mu_74 & 04140710 & Point Lonsdale, Australia & Human & 2004 & This study \\
\hline Mu_81 & 04149669 & Point Lonsdale, Australia & Human & 2004 & This study \\
\hline Mu_93 & 05131622 & St Leonards, Australia & Human & 2005 & This study \\
\hline Mu_05142109 & & East Gippsland, Australia & Possum & 2005 & This study \\
\hline Mu_JKD8170 & & Point Lonsdale, Australia & Possum & 2008 & This study \\
\hline Mu_08009899 & & Point Lonsdale, Australia & Human & 2008 & This study \\
\hline Mu_JKD8049 & & Point Lonsdale, Australia & Human & 2004 & This study \\
\hline \multicolumn{6}{|l|}{ M. marinum isolates } \\
\hline Mm_99/89 & & NSW, Australia & Human & 1994 & {$[34]$} \\
\hline Mm_99/84 & & Western Australia & Bilby (Macrotis lagotis) & 1999 & [34] \\
\hline Mm_99/87 & & Western Australia & Human & 1996 & [34] \\
\hline Mm_1726 & & Louisiana, USA & Armadillo & 1986 & [35] \\
\hline
\end{tabular}

*These isolates have been referred to by other species names as indicated but here are considered $M$. ulcerans and have been given the prefix "Mu_". Note: Additional sequencing and assembly data in Additional file 1: Table S1. 
clustering by homology searches. Reads and contigs were aligned to both the $M$. marinum $\mathrm{M}$ and $M$. ulcerans Agy99 reference chromosomes (Figure 1) and the $M$. ulcerans Agy99 pMUM001 plasmid (Figure 2).
To assess how well our collection represents the genetic diversity of the MuMC, we modelled the number of novel genes discovered with the addition of each new genome using a de novo assembly and an ortholog

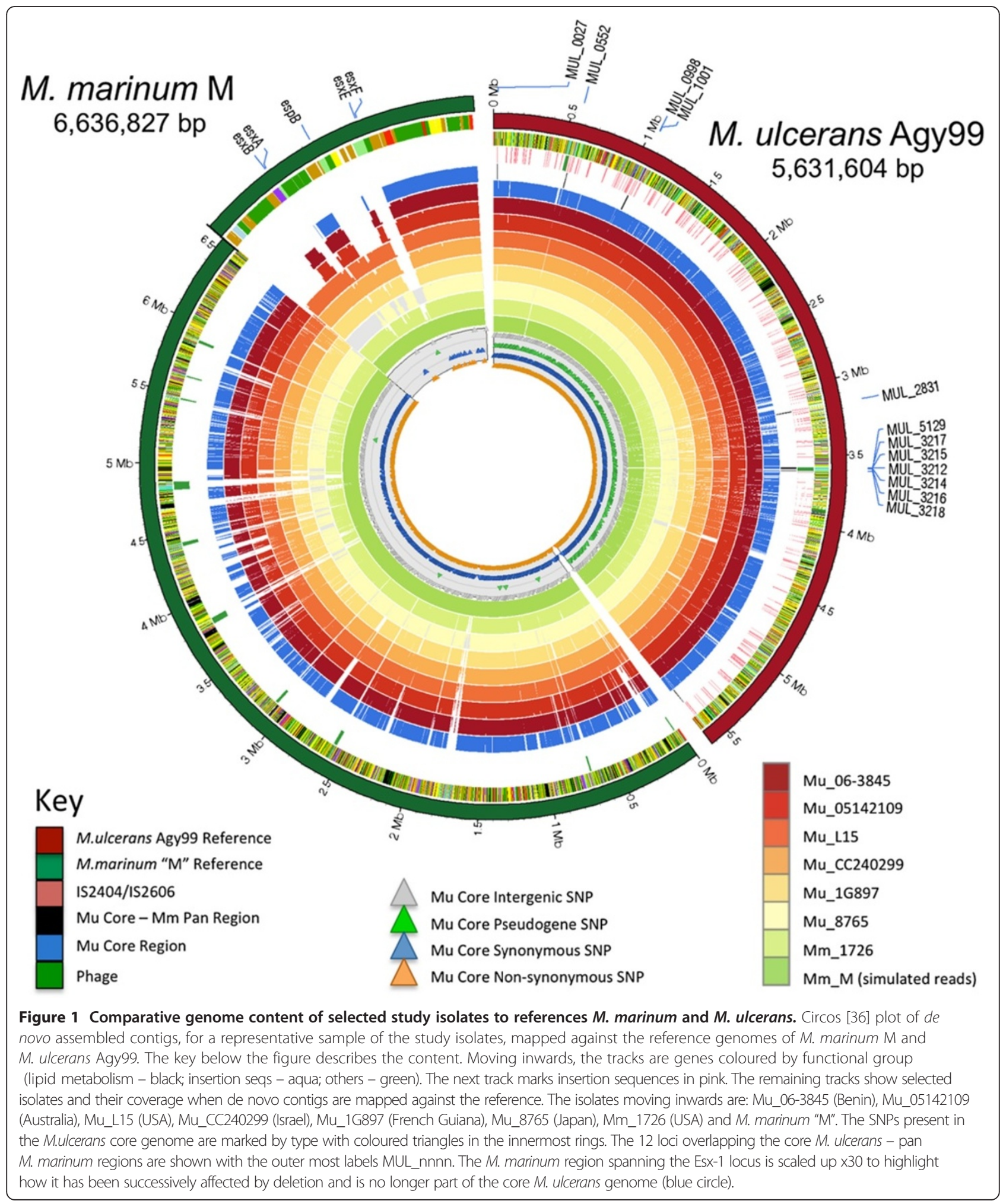




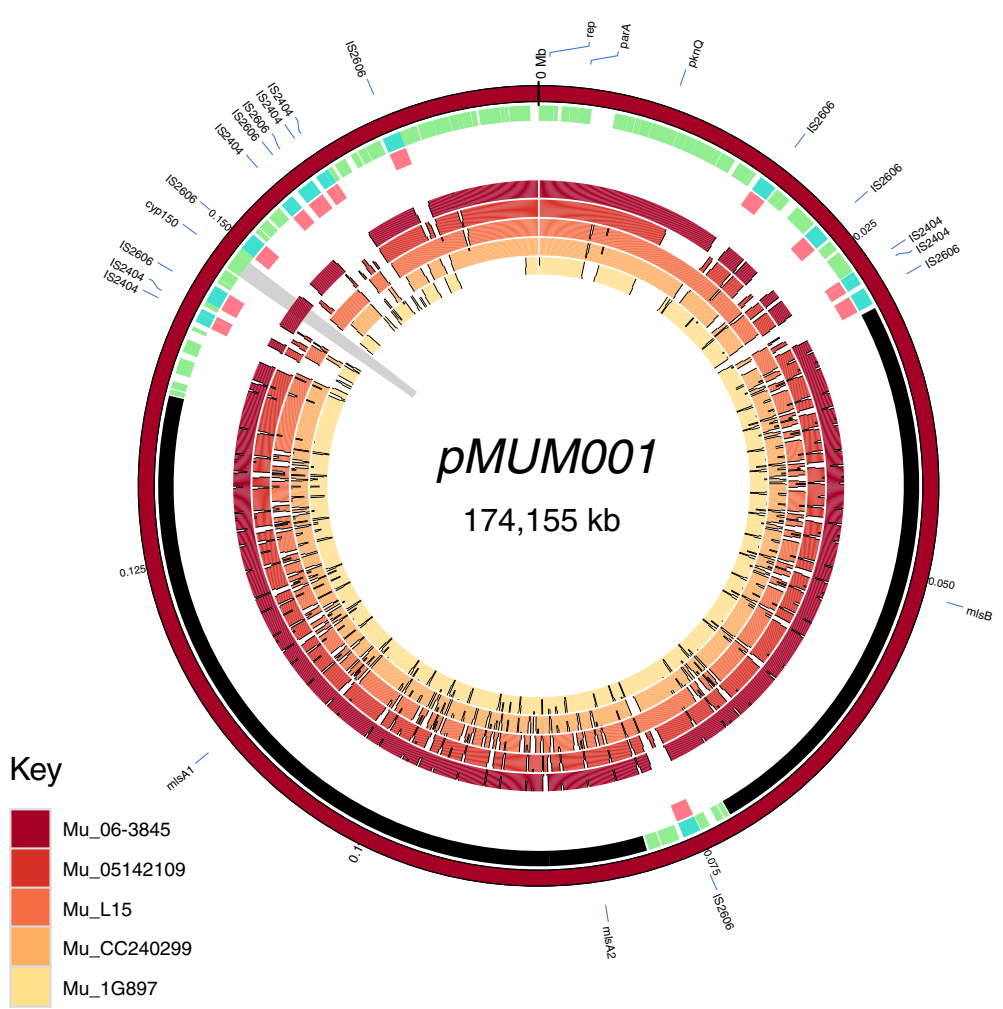

Figure 2 Comparative genome content of plasmid pMUM001. Circos [36] plot of de novo assembled contigs, for a sample of the study isolates, mapped against reference chromosome plasmid pMUM001. ISs and known genes are labelled in the outermost ring. Moving inwards, the tracks are genes coloured by functional group (lipid metabolism - black, insertion seqs - aqua, others - green). The next track marks insertion sequences in pink. The remaining tracks show selected isolates and their coverage when de novo contigs are mapped against the reference. The isolates moving inwards are: Mu_06-3845 (Benin), Mu_05142109 (Australia), Mu_L15 (USA), Mu_CC240299 (Israel), Mu_1G897 (French Guiana). The cyp150 gene (mup053) has been highlighted (grey wedge) to show its presence in African isolates and absence in other isolates.

clustering approach (see Methods). We assessed the trend by fitting a power curve to estimate the exponent, which is indicative of whether the pan genome is 'open' or 'closed' $\left(y=519.8 x^{-0.712}, R^{2}=0.99765\right)[37,38]$. Our MuMC isolates indicated an open pan-genome (exponent $>-1$ ), meaning that the total number of genes would continue to increase if more isolates were included (Figure 3). Based on the model, we predict that further sequencing within the MuMC would, on average, reveal fewer than 42 new coding sequences (CDS) per additional isolate. The core set of conserved orthologous clusters of CDS for our study isolates was found to be 3,305 CDS and modelling predicted that additional sequencing would result in no further reduction in core genome size.

\section{Mycolactone-producing mycobacteria form a monophyletic group}

The most sensitive method to identify a core set of conserved nucleotide positions among a set of genomes is via read mapping to a reference genome. The major advantages of this approach, compared to de novo assembly and ortho$\log$ clustering, are its independence from gene annotation and orthology and high (single nucleotide) resolution.
Hence we mapped sequencing reads from each isolate to the $M$. marinum $\mathrm{M}$ reference and defined the MuMC core genome as the set of $M$. marinum $\mathrm{M}$ nucleotide positions that were covered by at least three reads from every isolate. Repeat regions and regions spanning PE/PPE genes were excluded from this analysis due to ambiguous read mapping and poor coverage, respectively. We found a core of 4,362,138 bp (65.7\%) and 3,318 CDS of M. marinum M were present in all 35 mycobacteria of the complex.

Within this core genome, 128,463 variable nucleotide positions were identified among the sequenced MuMC genomes. These were randomly distributed around the chromosome (Figure 1) and were used to infer a distancebased neighbour joining phylogenetic tree (Figure 4). The tree topology confirms the previously inferred relationships between isolates by the lower-resolution MLST approach [21] and indicates that all mycolactone producing isolates (i.e. the MPM) belong to a single clonal group that diverged from a common $M$. marinum progenitor (Figure 4, root of red tree). In keeping with this observation, the MPM were relatively homogeneous with $0.06 \%$ median genome nucleotide divergence (interquartile range 0 $0.37 \%$ ) compared with M. marinum isolates with $0.86 \%$ 


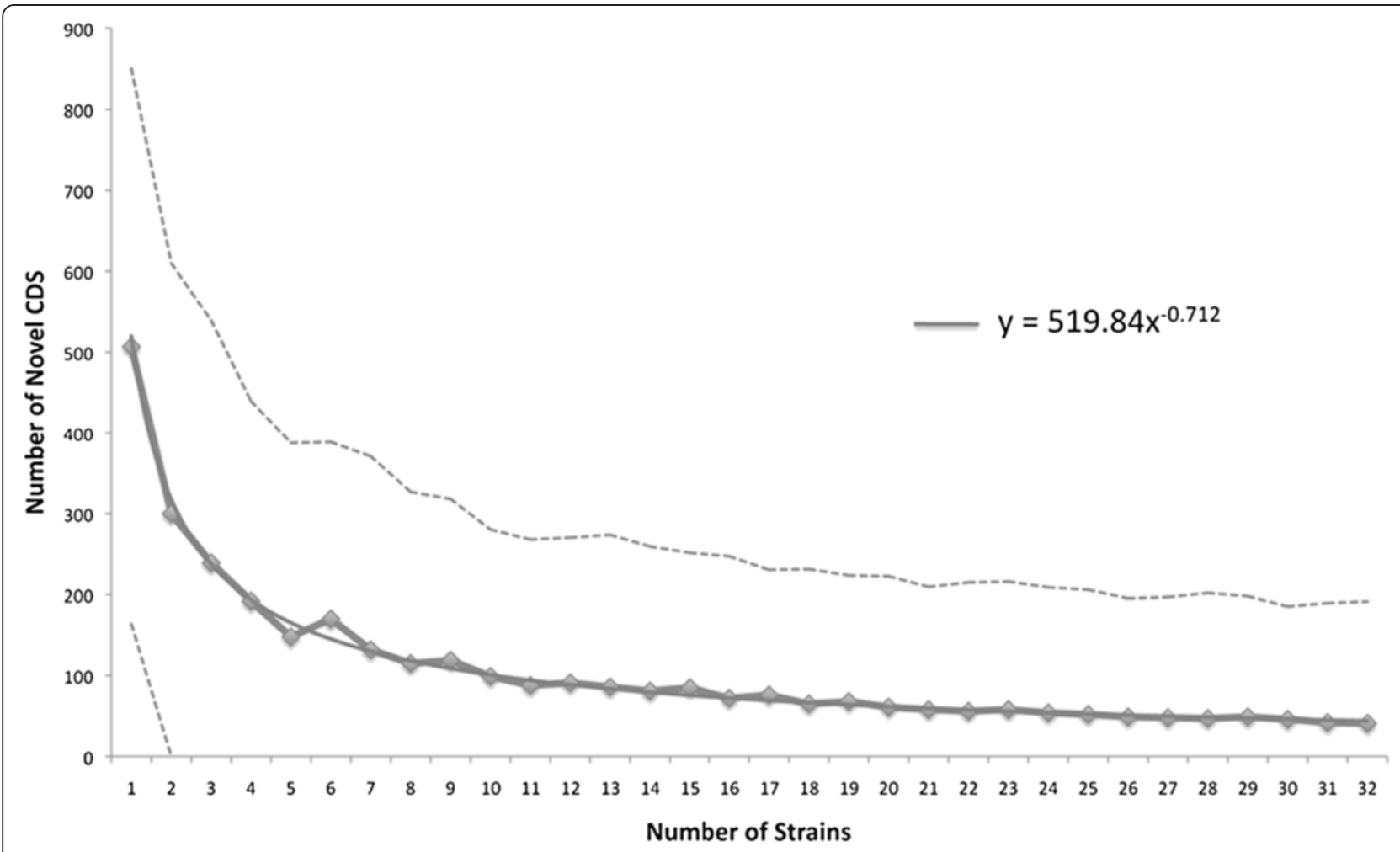

Figure 3 Open genome analysis. Median number of novel genes added per isolate (solid line) and the fitted power law model (equation inset). The median is for 1,000 random orderings of 32 of the study isolates. The dashed line shows the $+/-1$ standard deviation from the median.

nucleotide divergence $(0.59 \%-0.87 \%)$ (Figure 4$)$. This corresponds with genome-wide average nucleotide identity of 97\% among all genome pairs [39]. Within the MPM, at least three deep branching lineages were observed comprising, lineage 1: one human isolate from South America and globally distributed fish and frog isolates; lineage 2: a single $M$. ulcerans human isolate from Japan; and lineage 3: M. ulcerans human and other animal isolates from Africa and Australia. Lineage 3 corresponds to the previously reported "classical" MPM lineage, while the "ancestral" MPM lineage is refined here by lineages 1 and 2 [20]. The confirmation of a close genetic relationship between BUcausing MPM isolates from Africa and Australia (4,511 SNP differences) suggests that findings from studies of BU transmission in Australia may find corollaries in African $\mathrm{BU}$ endemic settings. Interestingly, there were even fewer nucleotide differences between some of the Lineage 1 isolates. For example M. pseudoshottsii and Mu_DL045, isolated two years apart from fish in the USA and Greece respectively, were separated by only 590 SNPs. Similarly, $\mathrm{Mu} \_\mathrm{CC} 240299$ and $\mathrm{Mu}$ _06-3844, isolated seven years and thousands of kilometres apart in Israel and Belgium, were separated by only 40 SNPs. These data indicate that clones of these mycolactone-producing ectotherminfecting mycobacteria are circulating worldwide. We also calculated the proportion of M. marinum and M. ulcerans reference genome sequence covered by each isolate of the MuMC (Additional file 2: Figure S2). The plot shows that MPM isolates are clearly differentiated from $M$. marinum by their gene content, with less than $90 \%$ of genomic sequence conserved between MPM and M. marinum isolates. In contrast to other pathogens such as Shigella, which has four main variants that have emerged independently [40], it appears that MPM have evolved only once through the acquisition of the virulence plasmid pMUM and IS expansion. Hence our data confirm both high genomic coherence and a common ancestry among all the MPM, supporting our previous argument that all MPM should be considered M. ulcerans [24]. Hereafter we refer to all MPM as M. ulcerans.

\section{Geographical restriction of $M$. ulcerans over short time scales}

Through our core genome SNP comparisons we were able to compare isolates within localised geographic regions. Using the same read-mapping approach as described above we defined a 5,190,533 bp core genome and phylogeny for the subset of 13 isolates from Benin and Ghana (Figure 5). Only 396 variable nucleotide positions were identified, with an average pairwise distance of 160 SNPs differentiating isolates between Ghana and 


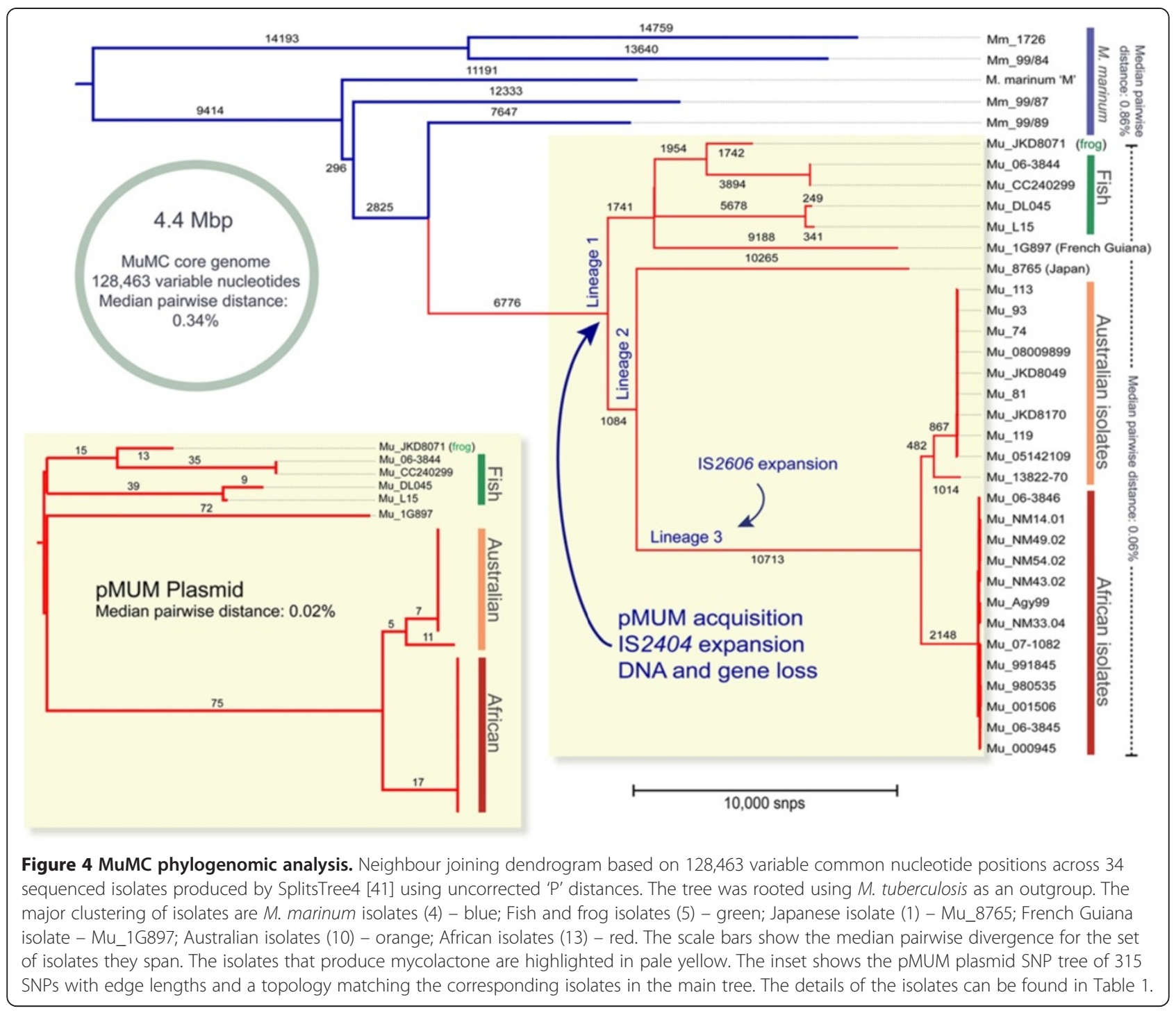

Benin. However, Mu_06-3846 isolated in the Couffo valley in Benin, clustered with the isolates from Ghana. The other six isolates from Benin originated from the Zou/Ouémé valley. A previous study has demonstrated a genetic difference between $M$. ulcerans from these two river basins [28]. An explanation for the apparent clustering of the Couffo strain $(\mathrm{Mu}$-06-3846) with the Ghanaian strains could be obtained by sequencing more isolates from the Couffo valley, from Togo (i.e. the area between the Couffo valley in Benin and Ghana) and Ghana. The population structure revealed in Figure 5 indicates localized clonal expansion in Ghana and Benin, suggesting that most $\mathrm{BU}$ infections in these areas result from local transmission of a single circulating clone, with only occasional transfer of clones between geographic areas. The environmental isolate $M$. ulcerans $\mathrm{Mu}$ _06-3845, obtained from an aquatic insect in Benin, clustered with the BU Zou/Ouémé Benin isolates but is distinct from a human isolate obtained from the same region at the same time $\left(\mathrm{Mu} \_001506\right)$. Among this limited set of isolates from the Zou/Ouémé valley in Benin, no finer-scale geographical clustering was observed. This is consistent with a previous study of isolates from an area of comparable size in Ghana [30].

We used the same approach to construct a phylogenetic tree for the mycolactone plasmid pMUM001. No pMUM001 sequences were detected among the four $M$. marinum isolates or $M$. ulcerans isolate $\mathrm{Mu} 8765$ (Lineage 2), indicating that the plasmid was absent from these isolates. $\mathrm{Mu} 8765$ has previously been reported to have lost its plasmid during laboratory passage [26]. All other MPM isolates carried the plasmid, confirming the central role of pMUM001 in the evolution of this complex. A total of 315 pMUM001 SNPs were identified among the MPM isolates, permitting construction of a distance-based phylogenetic tree for the plasmid 

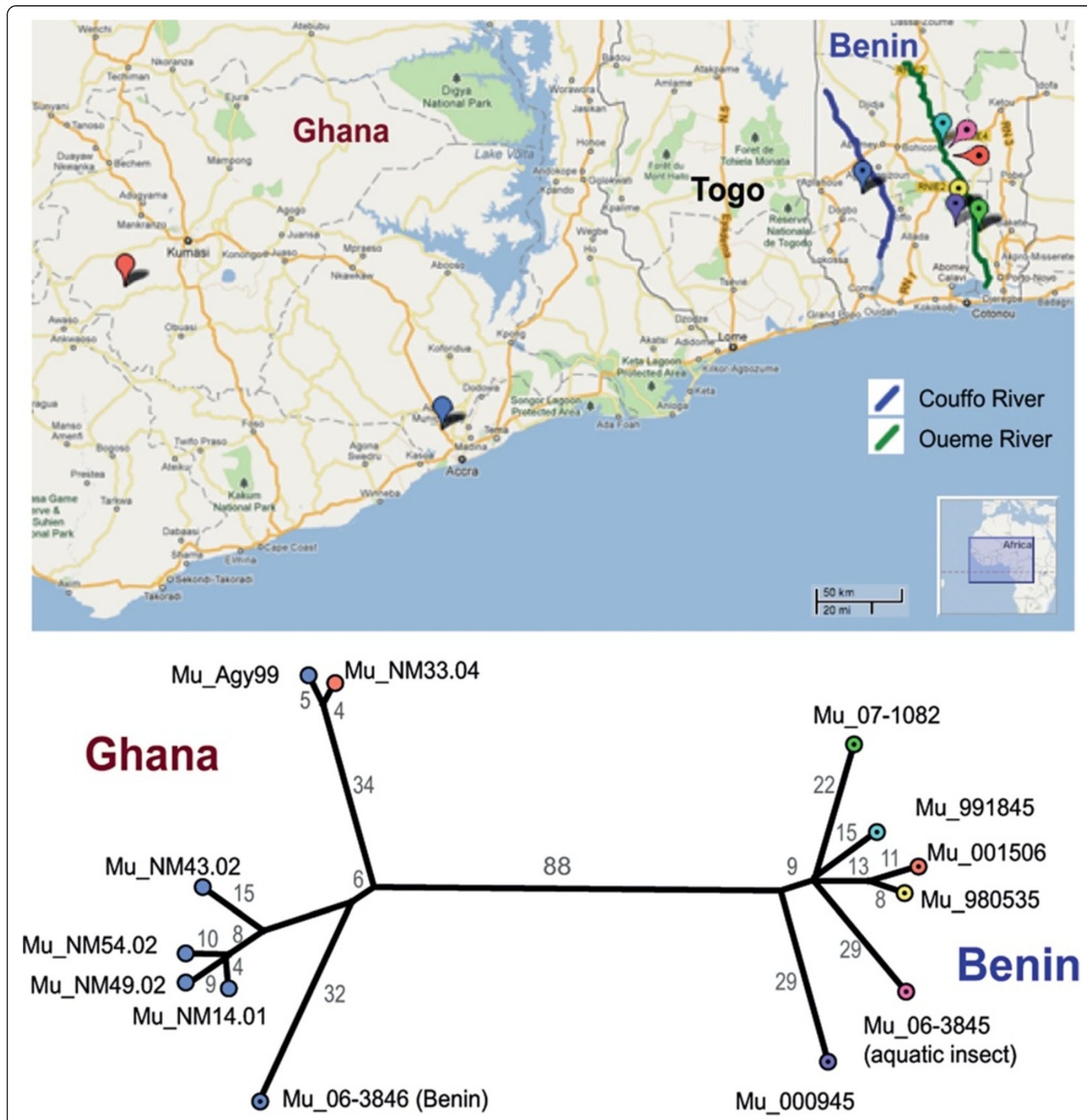

Figure 5 African isolates tree and map. Unrooted neighbour joining tree produced by SplitsTree4 [41] using uncorrected ' $P$ ' distances and based on 396 variable common nucleotide positions from a core of 5,190,553 bp among the 13 isolates from Ghana and Benin. The map shows their geographic distribution with pin colours corresponding to the tree. The isolate Mu_06-3846 is from Benin but clusters with the Ghanian isolates (see text). Map produced using Google Maps.

(Figure 4, inset). The plasmid phylogenetic tree closely matches both the topology and relative branch lengths of the core chromosome tree, consistent with coevolution of the plasmid and chromosome in each lineage [21]. By comparing read depths for plasmid and chromosome (see Methods), we estimated an average pMUM copy number of 1.8 copies per cell (range 1.3 for Mu_CC240299 to 3.1 for Mu_JKD8071).
Inferred genomic characteristics of $M$. ulcerans MRCA

First, we identified ancestral SNPs that differentiate the most recent common ancestor (MRCA) of M. ulcerans from the rest of $M$. marinum, which may have played a role in evolution of the distinctive phenotypic characteristics of $M$. ulcerans. Using M. marinum " $\mathrm{M}$ " as a reference, we identified 4,170 such SNPs (including small indels), spanning the entire chromosome and 
comprising 607 intergenic, 2,254 synonymous, 1,301 non-synonymous and eight SNPs occurring within pseudogenes (Figure 1, Additional file 3: Table S2). This list of mutations, which includes the recently reported hspR_2 regulatory mutation [42], will be important for research exploring functional characteristics of $M$. ulcerans. The identification of these SNPs is also the foundation for evaluation of evolutionary forces acting on specific genes (see later section).

Next, we investigated chromosomal regions that were conserved within $M$. ulcerans but absent from M. marinum. We identified 11 DNA segments of up to 2,688 bp in length, overlapping 15 CDS (total 10,256 bp) (Figure 6A). The locations of these CDS on the M. ulcerans chromosome are shown in Figure 1 and their annotations are described in Additional file 4: Table S3. These M. ulcerans-specific chromosomal genes include seven prophage genes from phiMU02, a phage-like polymerase (MUL_0027), a putative lipase (MUL_2832) and six hypothetical proteins. Hence other than the pMUM plasmid, ISs and phiMU02 as already described, $M$. ulcerans isolates have few other species-specific DNA elements and is instead characterised by gene loss. However the eight identified $M$. ulcerans-specific genes may be important for the ecology of $M$. ulcerans and warrant further investigation.

We also explored differences in gene content between M. ulcerans isolated from different geographic locations or from different hosts (Figure 6B, 6C, Additional file 5: Table S4). The core genome of $M$. ulcerans isolates known to infect humans comprised a strict subset of the core genome of fish and frog isolates. Nine CDS were found only in the fish/frog isolates and may be assumed to be superfluous for M. ulcerans infections in humans, although care needs to be exercised as there were only five fish and frog isolates on which to base this analysis. The products of the nine CDS include five hypothetical proteins, three putative hydrogenases and a putative oxononanoate synthase (BioF2_5). A total of 76 CDS were conserved in $M$. ulcerans lineages 1 and 2 but absent from lineage 3 (which contains only African and Australian $\mathrm{BU}$ isolates) (Figure 6C). These data suggest that the African and Australian BU isolates, which represent the majority of the global burden of BU infection in humans, have undergone further reductive evolution. This may be associated with additional adaptation to a more specialised niche, or genetic drift associated with passing through an evolutionary bottleneck. The 76 genes lost (Additional file 5: Table S4) include some involved in metabolic and respiratory processes, which may be no longer required in the more restricted environment occupied by lineage 3 isolates [43]. We also investigated novel $M$. ulcerans genes not present in the reference genome (Agy99, an African BU isolate from Lineage 3) via annotation and comparison of de novo assemblies of the novel isolates. The African and Australian isolates, also from Lineage 3, had no novel CDS apart from a putative integrase in six of the ten Australian isolates. The remaining $M$. ulcerans isolates from other lineages had many more novel CDS as follows; Mu_JKD8071 (128), Mu_06-3844 (93), Mu_CC240299 (93), Mu_L15 (13), $\mathrm{Mu} \_1 \mathrm{G} 897$ (8), Mu_8765 (3). The numbers of novel CDS largely reflects the isolates' similarity to the reference genomes as shown in Additional file 2: Figure S2.

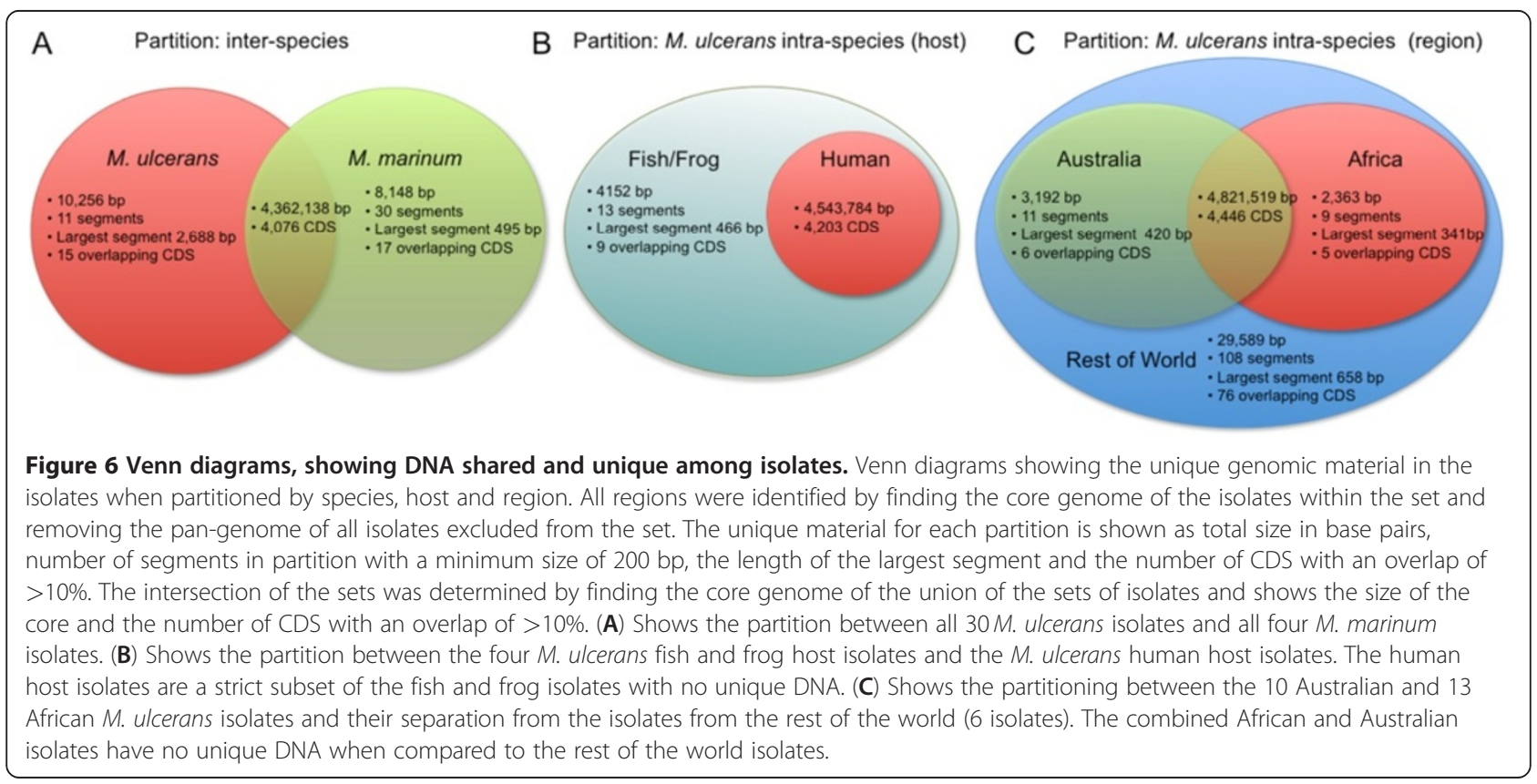


Figure 6 also shows the largest segments of DNA that could be used to discriminate between isolates by species, host and region (genomic locations are listed in Additional file 5: Table S4). For example, to discriminate between a $M$. ulcerans and a $M$. marinum isolate, there is a 2,688 bp segment that is present in all M. ulcerans isolates and absent from all other $M$. marinum isolates. Together with previously described deletions, these DNA segments could provide the basis of a set of DNA diagnostic tests to identify the species, host or region of an unknown isolate.

The M. ulcerans Agy99 reference contains the second highest percentage $(13.8 \%)$ of pseudogenes within a diverse set of 64 prokaryotic genomes [44]. M. leprae has the highest percentage (36.5\%) and represents an extreme case of genome reduction combined with loss of function. The pseudogenes present within the MRCA of the $M$. ulcerans isolates provide insights into the loss of function that may have been instrumental in the adaptation of $M$. ulcerans from the more generalist M. marinum. MRCA pseudogenes were inferred by assuming the inactivation of $M$. marinum CDS (intact in all $M$. marinum strains) via mutations in the M. ulcerans isolates affecting start codons, creating frame-shifts or introducing premature stop codons.
This analysis resulted in a list of 185 putative ancestral pseudogenes and/or deletions that were identified in all M. ulcerans, of which 83 were absent in Agy99 due to gene deletion (Additional file 6: Table S5). Hence at least $25 \%$ of the pseudogenes present in M. ulcerans Agy99 - or lost from this isolate by deletion - are shared by all extant M. ulcerans isolates and indicates that significant remodelling and adaptation through reductive evolution was occurring in the $M$. ulcerans MRCA before the global radiation of the species. The number of inferred pseudogenes and deleted genes per isolate is shown in Figure 7 and shows that lineage 3 strains have all lost substantially more coding potential than lineage 1 strains. An examination of the distribution of these ancestral pseudogenes by functional group shows that CDS belonging to one specific group, "cell wall and cell wall processes", are significantly overrepresented (35\%, compared with $\sim 22 \%$ for all CDS in M. marinum $\mathrm{M}$ and M. ulcerans Agy99). Table 2 summarizes some of the key CDS losses that may have played a role in the early adaptive responses of $M$. ulcerans following the acquisition of pMUM and a pathway of reductive evolution and includes loss of CDS required for synthesis of selenocysteine-containing proteins, anaerobic respiration, stress responses, intracellular

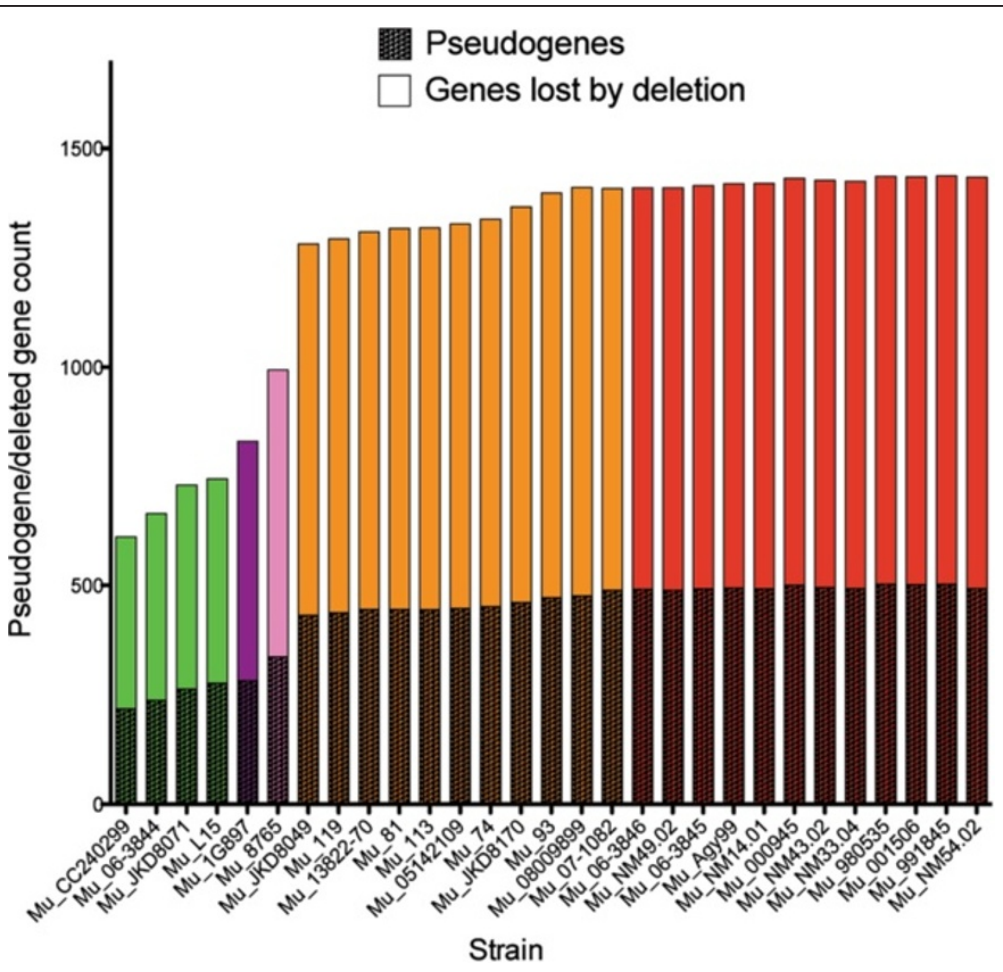

Figure 7 Inferred pseudogenes and deleted genes. Counts of inferred pseudogenes and deleted genes among the study isolates. The counts were inferred by analysis of SNPs that would render M. marinum reference CDS inactive and read coverage mapping showing partially or fully deleted CDS. The isolates are coloured by groups; African isolates - red, Australian isolates - gold, French Guiana isolate - purple, Japanese isolate - pink, fish and frog isolates - green. 
Table 2 Noteworthy genes or loci absent in the M. ulcerans MRCA

\begin{tabular}{|c|c|c|c|}
\hline Gene(s) & Locus_tag(s) & Comments & Reference \\
\hline \multirow[t]{3}{*}{ selA } & MMAR_5190 & selenocysteine synthase, required for the synthesis of proteins containing selenocysteine. & [1] \\
\hline & $\begin{array}{l}\text { MMAR_5194 } \\
\text { MMAR_5195 }\end{array}$ & $\begin{array}{l}\text { Formate dehydrogenase alpha and beta subunits, selenocysteine-containing. } \\
\text { Likely role in anaerobic growth. }\end{array}$ & \multirow[t]{2}{*}{ [1] } \\
\hline & MMAR_2615 & Anaerobic dehydrogenase (putative nitrate reductase), possible role in anaerobic growth. & \\
\hline lgt & MMAR_2416 & $\begin{array}{l}\text { Lgt acylates prolipoproteins, thus key role in lipoprotein synthesis. Lgt mutant of } \\
\text { S. aureus is less immunogenic. }\end{array}$ & {$[45-47]$} \\
\hline plcB_2, & MMAR_1485 & \multirow{4}{*}{$\begin{array}{l}\text { M. marinum has six plc genes. Four of these are absent in all M. ulcerans. Phospholipase C } \\
\text { enzymes can cause direct or indirect enzymatic hydrolysis of host cell membrane } \\
\text { phospholipids and appear important for mycobacterial intracellular survival. }\end{array}$} & \multirow[t]{4}{*}{ [48] } \\
\hline plcB_5, & MMAR_3656 & & \\
\hline plcB_6, & MMAR_4722 & & \\
\hline plcB_3 & MMAR_0284 & & \\
\hline $\operatorname{cst} A$ & MMAR_1616 & $\begin{array}{l}\text { Carbon starvation protein, CstA. In E. coli, cstA encodes a peptide transporter and is } \\
\text { induced by carbon starvation. Maybe part of a redundant stress response system in M. ulcerans. }\end{array}$ & [49] \\
\hline cueO & MMAR_1618 & $\begin{array}{l}\text { Multicopper oxidases protect against oxidative stress. This enzyme has functions in } \\
\text { tolerance to copper, and iron and manganese oxidation in a range of bacteria. It catalyses the } \\
\text { oxidation of cuprous copper, ferrous iron and diphenolic compounds. In Salmonella, a cueO } \\
\text { deletion mutant is less virulent in mouse model. Divergent transcriptional arrangement with cstA. }\end{array}$ & [50] \\
\hline$i d s B 1$, & MMAR_3212 & \multirow{3}{*}{$\begin{array}{l}\text { Catalyzes the trans-addition of three molecules of IPP onto DMAPP to form geranylgeranyl } \\
\text { pyrophosphate which is a precursor of the ether-linked lipids. Impact here of diverting } \\
\text { all isoprenoid biosynthesis to the non-mevalonate pathway is not known. Possibly more favourable } \\
\text { energetically if spending cellular resources on mycolactone synthesis. }\end{array}$} & \multirow[t]{3}{*}{ [1] } \\
\hline$i d s B 2$ & MAR_3219 & & \\
\hline idsA1 & MMAR_5095 & & \\
\hline
\end{tabular}

survival, acylation of lipoproteins and biosynthesis of isoprenoids.

\section{Impact of IS2404 and IS2606 on M. ulcerans genome architecture}

A key differentiator between $M$. marinum and $M$. ulcerans is the presence of multiple copies of IS2404 and IS2606 in M. ulcerans leading to genome plasticity and remodelling. We estimated the copy number of these IS in each isolate and confirmed that neither IS was present in any of the $M$. marinum isolates, as reported previously [1]. We found that $M$. ulcerans genomes from Lineages 1 and 2 had only $1-4$ copies of IS2606, while a massive expansion has occurred in Lineage 3 resulting in 63-98 copies per genome (Figure 8). Conversely, we identified a large number (41-81) of novel IS2404 insertions in Lineage 1 and 2 isolates (relative to the Agy99 reference) but only a single novel IS2404 insertion in 9 out of the 23 Lineage 3 isolates. These data confirm that ISs are continuing to modify the $M$. ulcerans genome. The sequence data of lineage 1 isolates Mu_CC240299 and Mu_06-3844 each contain a single copy of IS2606 that could be located within the pMUM plasmid but no copies were found within the chromosome (see Methods). As well as containing IS2404, the pMUM plasmid contains between one and eight copies of IS2606 and is therefore the likely origin of IS2404 and IS2606 in M. ulcerans chromosomes.

\section{M. ulcerans genes under selective pressure}

Identification of CDS that are under selective pressure can give additional insights into the specific nature of the environment(s) an organism encounters. The ratio $\mathrm{dN} / \mathrm{dS}$ is the number of non-synonymous substitutions per non-synonymous site $(\mathrm{dN})$ to the number of synonymous substitutions per synonymous site $(\mathrm{d} S)$; hence $\mathrm{dN} / \mathrm{dS}>1$ implies diversifying selection, while $\mathrm{dN} / \mathrm{dS}$ $<1$ implies purifying selection through loss of mutations that cause changes at the protein level. To compare selection in the $M$. marinum isolates against the $M$. ulcerans isolates, two likelihood function (LF) models were generated that compared a constant $\mathrm{dN} / \mathrm{dS}$ across all isolates against a model where $\mathrm{dN} / \mathrm{dS}$ differed across the tree. The dN/dS value across a core set of 2,379 CDS for the full tree was 0.181 (constant model) while the values for the differing sub-branches (varying model) were 0.093 for M. marinum isolates, 0.484 for African Lineage $3 \mathrm{M}$. ulcerans isolates, 0.515 for Australian Lineage $3 \mathrm{M}$. ulcerans isolates and 0.459 for M. ulcerans lineages 1-2. This is consistent with relaxation of purifying selection in $M$. ulcerans compared to M. marinum (log likelihood ratio statistic $=8988.6$, degrees of freedom $=63, p<1 \mathrm{e}$ $6)$, however the younger age of $M$. ulcerans could also explain this result [51]. This $\mathrm{dN} / \mathrm{dS}$ variation was consistent across all annotated functional groups: (M. ulcerans isolates $\mathrm{dN} / \mathrm{dS}$, M. marinum isolates $\mathrm{dN} / \mathrm{dS}$ ) conserved hypotheticals $(0.538,0.191)$, cell wall and cell processes $(0.524,0.165)$, lipid metabolism $(0.484,0.160)$, regulatory proteins $(0.470,0.160)$, intermediate metabolism $(0.469$, 


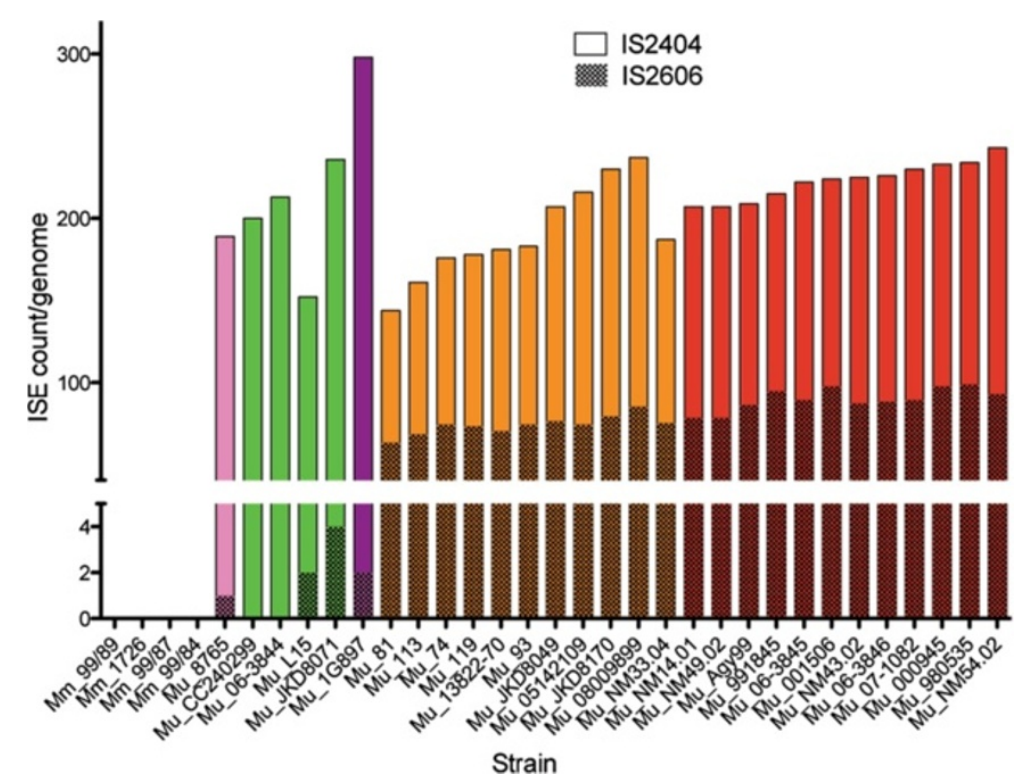

Figure 8 IS2404 and IS2606 distribution among isolates. Counts of IS2404 and IS2606 among the study isolates. The counts were inferred from coverage of reads mapping to each IS. The isolates are coloured by groups; African isolates - red, Australian isolates - gold, French Guiana isolate - purple, Japanese isolate - pink, fish and frog isolates - green. IS2404 and IS2606 were not detected in the non-MPM M. marinum isolates.

0.148), information pathways $(0.402,0.128)$. Next we compared $\mathrm{dN} / \mathrm{dS}$ at the level of individual CDS. Our model parameter start values were taken from the LF and the tree previously used to compare regions using the full set of CDS. This improved the ability of the new LF to be more rapidly and reliably optimised. Of the 2,379 CDS examined, $172 \mathrm{CDS}$ had a $\mathrm{dN} / \mathrm{dS}>1$ for the M. ulcerans isolates (Additional file 7: Table S6). This set was dominated by two functional groups, 'cell wall and cell processes' (44 CDS) and 'conserved hypotheticals' (56 CDS). Notable CDS in this set of genes under diversifying selection included esxE and es $x F$ that encode WXG100 domains and are orthologs of the secreted T-cell antigens EsxA and EsxB. The stress response sigma factor $\operatorname{sig} M$ was also identified as under diversifying selection, perhaps indicating remodelling of the SigM regulon in M. ulcerans [2] (Figure 1). In $M$. tuberculosis, sigM appears to positively regulate esxE and esxF expression while negatively regulating certain non-ribosomal peptide synthetases and polyketide synthase genes involved in biosynthesis of cell wall lipids [52,53]. Lipoproteins, another class of cell wall-associated molecule, also featured among the genes displaying evidence of diversifying selection $(\operatorname{lpp} N$, $l p p D, \operatorname{lpr} A$ ) and perhaps links to the predicted loss of Lgt activity and the ability to acylate prolipoproteins (Table 2).

We also examined CDS that contained only nonsynonymous SNPs, prohibiting calculation of a $\mathrm{dN} / \mathrm{dS}$ ratio, or were not core to $M$. ulcerans, and compiled a list of additional CDS with an overabundance of nonsynonymous SNPs, sorted by the difference between non-synonymous and synonymous SNPs (see Methods). The 50 most variable proteins mirrored the dominant functional groupings of the high $\mathrm{dN} / \mathrm{dS}$ analysis (Additional file 7: Table S6), including esxE/F as well as enoylCoA hydratase echA4_2 and ssp. Ssp (signal peptide protease IV) is a serine protease required for digestion of cleaved signal peptides, indicating that M. ulcerans may be under pressure to modify their secretome. Also of interest is $m y m T$, encoding a copper-binding protein, a cytoplasmic metallothionein and in M. tuberculosis, sequesters excess copper in the bacterial cell [54].

The close relationship between $M$. tuberculosis and the MuMC led us to investigate whether the orthologous human $\mathrm{T}$ cell epitopes for $M$. tuberculosis were conserved in $M$. ulcerans. The genes encoding antigens in human pathogens tend to be under diversifying selection in order to evade host immunity and it has been suggested that this is not the case with $M$. tuberculosis [55]. We found 20 MuMC orthologs (>80\% AA identity) of the 86 human T-cell antigens in $M$. tuberculosis H37Rv (Additional file 8: Table S7). These orthologs all had very low $\mathrm{dN} / \mathrm{dS}$ ratios ( $M$. marinum isolates median 0.05 , range $0.15, M$. ulcerans isolates median 0.17, range 0.95) suggesting that these CDS are under purifying selection in the MuMC as they are in $M$ tuberculosis. 


\section{Discussion}

Whole genome sequencing and comparison of this large collection of isolates has shown that all mycolactoneproducing mycobacteria (collectively referred to in this study as $M$. ulcerans) evolved from a common $M$. marinum progenitor by a combination of horizontal gene transfer (pMUM and phage), IS-mediated deletion and point mutation (Figure 4). These evolutionary processes resulted in extensive gene loss (185 CDS), changes in gene expression (e.g. hsp 18 and possibly sigM) and likely changes in gene function through positive selection, establishing M. ulcerans as a highly specialized, nicheadapted mycobacterium. Our data indicate these changes were followed by global dispersal of $M$. ulcerans and further diversification and adaptive evolution.

Scrutiny of the types of genes gained, lost or modified in conjunction with other experimental evidence provides some clues regarding the nature of the niche environment in which the M. ulcerans MRCA was able to flourish and in which today's isolates still survive. Foremost among the DNA gained was in the pMUM plasmid. The acquisition of this plasmid occurred early in the evolution of $M$. ulcerans as demonstrated by the congruent tree topologies inferred from chromosome and plasmid sequence alignments (Figure 4). The synthesis of mycolactone, a potent immunosuppressive small molecule encoded in the pMUM plasmid, presumably gave a population of $M$. marinum cells the ability to persist in a place their generalist relatives could not. In previous research we have described how recombination and gene conversion has shaped the unusually repetitive gene structure of the mycolactone PKS, such that the $110 \mathrm{~kb}$, three-gene PKS locus on pMUM comprises only $10 \mathrm{~kb}$ of unique sequence. This unusual gene structure and its resultant instability are strongly suggestive of intense selection acting on $M$. ulcerans populations to maintain mycolactone production [5,56,57].

The loss of the mevalonate pathway for synthesis of isoprenoid lipids was originally observed in the genome of M. ulcerans Agy99 [1]. In the current study we show that this trait was established in the M. ulcerans MRCA and was probably a key adaptive response of the bacterium following the acquisition of pMUM (Table 2). It is possible that loss of this metabolic capacity freed essential resources for critical mycolactone synthesis or alternatively that these pathways and metabolites were redundant in the niche environment occupied by $M$. ulcerans. Other potentially significant gene losses in $M$. ulcerans include selenocysteine synthase (selA), required for the synthesis of proteins containing selenocysteine, and the linked genes encoding the alpha and beta subunits of a putative selenocysteine-containing formate dehydrogenase, with a possible role in anaerobic growth; mutations that suggest the M. ulcerans MRCA lost the ability to grow anaerobically.

It is also striking that genes associated with the intracellular lifestyle of mycobacterial pathogens such as $M$. marinum and M. tuberculosis have been lost in M. ulcerans. Genes predicted to be inactive include four phospholipase enzymes (PlcB_2,3,5\&6) and сиеO (Table 2). As well as playing roles in intracellular replication in other bacteria, these CDS are also components of the bacterial cell wall. There appears to have been significant selective pressure on $M$. ulcerans to reduce or change its cell wall and cell surface antigenic profile. In this respect another noteworthy pseudogene conserved in M. ulcerans is lgt (MUL_1594). Lgt is a prolipoprotein diacylglyceryl transferase that acylates prolipoproteins at a conserved N-terminal cysteine [45]. An lgt mutation in Staphylococcus aureus causes growth rate attenuation, an accumulation of prolipoproteins in the culture supernatant, and reduced activation of innate immune responses [46,58]. The loss of lgt in $M$. ulcerans might therefore lead to aberrantly or non-acylated lipoproteins with reduced immunogenicity, like the $S$. aureus mutant.

The modification of the cell wall appears to have continued in the BU-associated M. ulcerans lineage 3 genomes, which contain an additional 589 pseudogenes or deleted regions of which $30 \%$ are predicted to have encoded antigens or cell wall associated proteins, including EsxA_2, EsxA_3, and Hspx_1. The deep branching lineage and clonal nature of the African and Australian lineage 3 isolates, which are most commonly involved in human infections, have the signature of passing through a second evolutionary bottleneck: gene deletions, further loss of gene function, chromosomal rearrangements and the expansion of another IS (IS2606 from the pMUM plasmid). Each of the M. ulcerans lineages probably represents different ecotypes, reflecting adaptation to related but distinct niche environments. It may be that each lineage is best described as an $M$. ulcerans ecovar.

We tried to identify a temporal signal in our sequence data to estimate divergence dates for particular lineages of the MuMC. However, while our phylogenetic inferences were highly robust (100\% bootstrap values for major branches of our tree, Figure 4) no linear correlation between branch length and year of isolation was observed. We suspect there is variation in the effective number of generations per year across the complex, perhaps related to different niches, reservoirs or modes of living. Such variation has recently been observed in M. tuberculosis [59]. The lack of a temporal signal in these data raises doubts around previous estimates of divergence time that have assumed a constant molecular clock rate among the lineages of the complex $[26,34]$. 
However, sequence comparisons did reveal a compelling correlation between genes undergoing positive selection - as revealed by $\mathrm{dN} / \mathrm{dS}$ analysis - and those CDS inactivated or deleted (Additional file 6: Table S5, Additional file 7: Table S6), with mutations in all these groups skewed towards CDS involved in cell wall and lipid biosynthesis. These patterns point to significant selective pressures acting on $M$. ulcerans populations to devote resources (substrate and energy) towards the synthesis of mycolactones and modification of cell wall structures. Intriguingly, many of the cell wall metabolites lost via mutation are known to be highly antigenic in other mycobacteria. One interpretation of these observations is that the bacteria are responding to pressures from a host immune system, a point argued in a previous study [60]. Furthermore, when one considers that mycolactone is a potent immune suppressor with reported specificity for a mammalian microRNA that controls T-cell chemotaxis [61], this in turn leads to the idea that the niche occupied by $M$. ulcerans is a higher organism with a complex immune system. The discovery that Australian possums inhabiting BU endemic areas are susceptible to BU disease and harbour large number of $M$. ulcerans in their gastrointestinal tracts is consistent with this idea [14]. Nevertheless, these arguments are not consistent with the significant lack of variation seen among $M$. ulcerans proteins with putative T-cell epitopes (Additional file 8: Table S7), where an immune escape hypothesis would predict hypervariability not hyperconservation in these regions.

This study has also reinforced the close relationship between isolate origin and genotype for M. ulcerans strains that cause Buruli ulcer, notably those from Africa and Australia, where multiple isolates from one region were sequenced. The complete resolution of strain differences afforded by whole genome sequencing has shown how the genotype of $M$. ulcerans strains from two African countries correlate with place of origin. Isolates from the east of Benin are distinct from isolates in the West of the country or from a different country (Ghana, Figure 5). These findings suggest - as also demonstrated in a previous study in Ghana - that $M$. ulcerans transmission and microevolution generally occurs at a local level and therefore the source of the bacterium is somewhat fixed within a local region [30]. This observation should guide our thinking regarding the source of the bacteria in BU endemic areas, indicating that animal reservoirs of $M$. ulcerans are unlikely to be highly mobile. Yet, one should also consider that the relative paucity of genomic differences between isolates from Ghana and Benin also reflects the relatively recent spread of the bacteria across this entire region. Efforts to establish the rate of mutation of these isolates or genome analysis of a more temporally and spatially diverse collection of isolates from this region might help estimate the amount of time $M$. ulcerans has been extant in West Africa.

\section{Conclusions}

This study has given us our most comprehensive insight yet into the $M$. ulcerans-M. marinum complex. The isolates examined in the study covered a wide geographical diversity, and while future studies should include $M$. ulcerans isolates from other endemic countries together with more $M$. marinum isolates, whole genome comparisons of 35 isolates across the genetic diversity of the complex have yielded nucleotide-level granular detail of each isolate and their relatedness to each other. We have shown that all MPM are a single lineage whose divergence from $M$. marinum was characterised by acquisition of the pMUM plasmid, which conferred the ability to synthesise mycolactones (largely responsible for the immunomodulatory properties of $M$. ulcerans) and also introduced IS2404 and IS2606 into the chromosome; this was then followed by IS2404 and IS2606 expansion and extensive loss and modification of gene function, consistent with an evolutionary bottleneck and adaptation to a new niche. Based on these shared features we propose that all members of this lineage should be considered $M$. ulcerans. Furthermore, we suggest that $M$. ulcerans sublineages (such as the three we have identified in this study) should be considered M. ulcerans ecovars.

Examination of the classes of genes lost and modified in the M. ulcerans MRCA suggests a bacterial population occupying a niche environment that is aerobic, osmotically stable, dark (at least for lineage 3 isolates as shown by loss of UV-protecting pigment genes in a previous study) and possibly extracellular given the number of genes known to be involved in intracellular survival lost from M. ulcerans $[1,13,21]$. Support for an extracellular niche is also found in two separate studies showing M. ulcerans elaborates a mycolactone-rich extracellular matrix and specifically expresses a surface protein that promotes adherence during initial stages of biofilm formation $[42,62]$. Furthermore, it is difficult to ignore the striking depletion of immunogens and modification of cell-wall proteins in $M$. ulcerans that may be subject to interactions with the immune defences of a host organism.

This study has clarified our understanding of the origins of an emerging human pathogen, provided important insights into the search for the reservoir(s) of this pathogen and generated a significant resource for future research. It is clear that $M$. ulcerans is not a generalist, saprophytic mycobacterium but a highly specialized occupant of a protected niche. This concept should be considered in ongoing research efforts to pinpoint the 
reservoir(s) of $M$. ulcerans and to understand the transmission of BU.

\section{Methods}

Several bioinformatics approaches were used to analyse the sequence data. The workflow we developed is summarised in Additional file 9: Figure S1 and involved analysis of sequence differences and similarities between all strains. A mapping-to-reference approach was applied as well as de novo assembly of the sequenced reads. A summary of the isolate data is presented in Additional file 1: Table S1, including the number of reads and the de novo assembly statistics for each strain.

\section{Bacterial isolates and sequencing}

The isolates of $M$. ulcerans and $M$. marinum used in this study are described in Table 1. Genomic DNA was prepared from at least $50 \mathrm{mg}$ (wet weight) cell pellets, harvested from mycobacteria that were cultivated on egg-yolk agar media as previously described [42].

Genome sequencing of the isolates (except for $\mathrm{Mu}$ _DL045) was performed using an Illumina GAIIx DNA sequencer with 36,76 , or 100 cycle paired-end chemistry, generating read lengths of 35-101 nucleotides and 16-240x mean read depth per isolate. Sequencing statistics are provided in Additional file 1: Table S1. Mu_DL045 was sequenced using the Ion Torrent platform to generate single-ended reads. Read data for the study isolates $\left(\mathrm{Mu} \_06-3845, \mathrm{Mu} \_06-3846, \mathrm{Mu} \_07-1082\right.$, $\mathrm{Mu} 113, \mathrm{Mu} \_119, \mathrm{Mu}$ 74, Mu_81, Mu_93, Mu_05142109, Mu_JKD8170, Mu_08009899, Mu_JKD8049) have been deposited in the NCBI Sequence Read Archive (SRA) under accession SRP004497 [63].

Prior to further analysis, reads were filtered to remove those containing ambiguous base calls, any reads $<24$ nucleotides in length and reads containing only homopolymers. All reads were trimmed from the 3' end removing bases with the Illumina Read Segment Quality Control Indicator "B".

\section{Read mapping}

An in-house Python utility called Nesoni, which uses SHRiMP2 [64] for read mapping, was used to map novel sequence reads to reference genomes [65]. Nesoni identified SNPs and indels up to $\sim 10 \mathrm{bp}$ and predicted the consequences of SNPs and indels on CDS, e.g. frameshifts, premature stop codons and nonsynonymous codon changes. Five reference genomes and fragments were used for read mapping, (sequence name, Genbank accession number) (M. ulcerans Agy99, CP000325), (M. marinum strain "M", CP000854), (pMUM001 plasmid, BX649209), (pMUM002 plasmid, EU271968) and ( $p M U M 003$ plasmid, EU271967). To facilitate comparison of $M$. marinum and $M$. ulcerans reference genomes using Nesoni, short read data for M. marinum " $\mathrm{M}$ " was simulated via in silico shredding of the reference genome to generate a set of $100 \mathrm{bp}$ short reads (read depth x15 insert length $280 \mathrm{bp}$ ) using a utility function available within Nesoni. Average read depth and the number of SNPs detected by mapping to M. ulcerans Agy99 and M. marinum M are given in Additional file 1: Table S1.

The number of reads mapping to unique regions of the plasmids was used to infer plasmid copy number. The ratio of the mode of read depth for the reference plasmid (pMUM002 for Mu_06-3844, Mu_CC240299, $\mathrm{Mu} \_J$ D8071, Mu_L15, Mu_1G897 and pMUM001 for all other M. ulcerans strains) was compared to the mode of read depth for the chromosome.

\section{SNP analysis}

Variant sites (SNPs and indels) identified using Nesoni were concatenated to form a multiple alignment, from which a phylogenetic tree was constructed using SplitsTree4 (neighbour-joining tree based on uncorrected $\mathrm{P}$ distances) [66]. All major bifurcations had $>95 \%$ bootstrap support based on 1,000 runs. The tree shown in Figure 4 is based on SNPs identified using $M$. marinum $\mathrm{M}$ as the reference, however the same tree was recovered when using M. ulcerans Agy99 as the reference. SNPs were classified by their position on the annotated references of M. ulcerans Agy99 and M. marinum " $\mathrm{M}$ " and identified as 'intergenic', 'coding' or 'pseudogene'; 'coding' SNPs were further divided into synonymous, non-synonymous or nonsense SNPs. All SNPs called within annotated ISs or PE/PPE genes were excluded from SNP analysis due to the unreliability of read mapping in repetitive regions $[8,67]$.

\section{De novo assembly of short-read sequences}

Genomes were assembled using Velvet [68] with the help of VelvetOptimiser [69] to select appropriate parameter values for k-mer size, expected coverage and coverage cutoff. The Velvet assemblies yielded an average N50 of 18,399 bp; assembly statistics are given in Additional file 1: Table S1. Repetition of hundreds of copies of IS2404 and IS2606 within the M. ulcerans isolates was problematic and restricted the contig lengths, as the size of sequenced DNA fragments $(\leq 240 \mathrm{bp})$ was much smaller than the length of the ISs (1365-1444 bp). To identify IS disrupted contigs, paired reads were mapped to consensus IS2404 and IS2606 sequences. Where a read was mapped to the IS but its partner read was not, the unmapped partner reads were pooled and de novo assembled to identify the DNA sequences that flank the IS sequences. This analysis demonstrated that $51 \%$ ( $11 \%$ s.d.) of the contig breakpoints terminated at either IS2404 or IS2606. Mycobacterial genomes contain 
hundreds of PE/PPE genes, which pose problems for sequencing, read mapping and assembly due to their repetitive, low $\mathrm{G}+\mathrm{C}$ content 3 ' sequences [70]. Hence PE/ PPE genes were excluded from most downstream analysis including SNP calling and gene content analysis. Contigs were annotated by Prokka, an in-house bacterial genome annotation pipeline using Glimmer, EasyGene, Orpheus and GeneMark.

\section{Core and pan genome analysis}

The core genome was defined as all positions in the reference genomes that were covered by $\geq 3$ reads in all isolates; this information was derived from depth plots generated by Nesoni. The set of genomic material conserved in one set of isolates, A, but absent from a second disjoint set of isolates, $\mathrm{B}$, was obtained by taking the core genome of A minus (all positions conserved among A) and removing any bases present in $\mathrm{B}$.

To define the pan genome, CDS annotated in the reference or de novo assembly contigs were subjected to ortholog clustering. CDS were translated to amino acid sequences and clustered using CD-Hit [71] with a 70\% amino acid sequence identity threshold. A core set of $M$. ulcerans CDS was defined by identifying all clusters containing a $M$. ulcerans reference CDS and one or more CDS fragments, with a combined length within $15 \%$ of the reference sequence, from each novel $M$. ulcerans genome.

To assess the completeness of the set of study isolates, we calculated the median number of new genes added to the pan-genome for every additional genome, using 1,000 permutations of the study isolates ( $M$. ulcerans and $M$. marinum). A trend was modelled by fitting a power curve to estimate the exponent, indicating whether the pan genome is 'open' or 'closed' $[37,38]$.

\section{$\mathrm{dN} / \mathrm{dS}$ analysis}

The relative rates of change at synonymous and nonsynonymous sites $(\mathrm{dN} / \mathrm{dS})$ within protein coding regions was calculated using the BioPerl module Bio::Align:: DNAStatistics and the PyCogent toolkit [72]. The dN/dS analysis was performed for both individual CDS (using BioPerl and PyCogent) and for aggregate concatenated CDS (using PyCogent). To test for selection across multiple isolates using multiple CDS, analysis was restricted to an alignment of DNA sequences from all CDS that were present, without a premature stop codon, in all isolates $(2,379 \mathrm{CDS})$. This alignment, together with an unweighted neighbour-joining tree of the isolates, was provided as input to PyCogent modules that calculated the $\mathrm{dN} / \mathrm{dS}$ values across subsets of the isolates. The CNF codon substitution model was used for all analyses [72]. A representative set of study isolates was used: $M$. marinum Mm_1726, Mm_99/84, Mm_99/87, Mm_99/89; $M$. ulcerans lineage 1 Mu_CC40299, Mu_JKD8071, Mu_DL045, Mu_1G897; M. ulcerans lineage $2 \mathrm{Mu} 8765$; M. ulcerans lineage $3 \mathrm{Mu} 05142109, \mathrm{Mu}$ _Agy99. This alignment, together with the corresponding subset of the tree and a codon substitution model, was used to create a likelihood function (LF) which models selection using the PyCogent framework [73]. By specifying how the $\mathrm{dN} / \mathrm{dS}$ parameter values of the model vary, optimisation of the LF can estimate $\mathrm{dN} / \mathrm{dS}$ across the whole tree, a sub branch of isolates or individual isolates. The $\mathrm{dN} / \mathrm{dS}$ metric cannot identify CDS that contained only nonsynonymous SNPs but have no synonymous SNPs. As an alternative method of identifying CDS with an overabundance of non-synonymous SNPs, we calculated the average difference between the number of nonsynonymous and synonymous SNPs normalised for the length of CDS. Functional groups used to classify CDS were defined by the reference genome annotations and based on COG v1.0 / CDD groupings.

\section{Pseudogene identification}

In order to identify the pseudogenes in the M. ulcerans MRCA we examined the CDS SNP changes that were common in all $M$. ulcerans isolates but not present in any $M$. marinum isolates. All PE/PPE, ISs and M. marinum pseudogenes were excluded from this analysis. The remaining $M$. marinum CDSs were examined to determine whether the SNPs called by read mapping the $M$. ulcerans isolates onto $M$. marinum resulted in a change to the CDS that would likely render it inactive within the $M$. ulcerans isolates; namely (i) indels that result in frameshifts, (ii) premature stop codons truncating encoded protein, (iii) a change to the start codon, (iv) an IS insertion or (v) a deletion of part or whole of the CDS.

\section{Additional files} Additional file 1: Table S1 Strain table and summary statistics.
Isolates used in this study with sequencing summary statistics.

Additional file 2: Figure S2 Percentage DNA difference between isolates. Scatter Plot showing the percentages of DNA missing from references M. marinum " $\mathrm{M}$ " ( $\mathrm{y}$-axis) and M. ulcerans Agy99 and pMUM001 plasmid (x-axis). The percent missing is calculated by taking the number of zero coverage positions in the short read mapping to reference and dividing by total length of the reference. The dotted lines show the percentage missing from either reference to distinguish the $M$. marinum isolates from the MPM isolates. The clusterings are coloured as follows with the number in each cluster in brackets; M. marinum isolates (5) blue, Fish and frog isolates (5) - green, Japanese isolate (1) - pink, French Guiana isolates - purple, Australian isolates (10) - gold, African Isolates (13) - red

Additional file 3: Table S2 Core SNPs. Listing of core SNPs and short indels common to all $M$. ulcerans isolates when mapped to references $M$. ulcerans Agy99 and M. marinum M.

Additional file 4: Table S3 $M$. ulcerans specific CDS and features. List M. ulcerans Agy99 reference annotated features found in all $M$. ulcerans isolates but not found in any of the M. marinum isolates. 
Additional file 5: Table S4 Diagnostic regions. List of nucleotide diagnostic regions distinguishing strains between various strain groups: M. marinum strains, M. ulcerans strains, African strains, Australian strains, strains from other regions, human host strains and fish or frog host strains.

Additional file 6: Table S5 Ancestral Pseudogenes. List of putative ancestral pseudogenes.

Additional file 7: Table S6 High dN/dS CDS and CDS with high nonsynonymous SNPs. List of core M. marinum CDS with $\mathrm{dN} / \mathrm{dS}>1.0 \mathrm{in}$ representative M. ulcerans isolates (Mm_1726, Mm_99/84, Mm_99/87, Mm_99/89, Mu_CC40299, Mu_JKD8071, Mu_DL045, Mu_1G897, Mu_8765, Mu_05142109, Mu_Agy99).

Additional file 8: Table S7 $M$. marinum orthologs of $M$. tuberculosis T-cell antigens. List of $M$. tuberculosis T cell antigens with orthologs in $M$. marinum ' $M$ ' and M. ulcerans Agy99. Orthologs have $>80 \%$ amino acid identity.

Additional file 9: Figure S1 Work Flow. Schematic of the workflow with intermediate stages of the data shown in blue boxes. The inputs to the process are shown in red and comprise the annotated reference genomes and the short read sequencing data of the study isolates. The results are shown in green and include the phylogeny of the complex, novel CDS within each of the isolates, core and accessory genomes of the complex and putative ancestral pseudogenes.

\section{Abbreviations}

ANI: Average nucleotide identity; BU: Buruli ulcer; CDS: Protein-coding DNA sequence; Indel: Insertion or deletion in genome; IS: Insertion sequence; LF: Likelihood function; MLST: Multi-locus sequence typing; MPM: Mycolactone producing mycobacteria; MRCA: Most Recent Common Ancestor; MTBC: Mycobacterium tuberculosis complex; MuMC: Mycobacterium ulcerans-Mycobacterium marinum complex; N50: The length of the contig such that $50 \%$ of the assembly is contained in contigs of length N50 or greater.

\section{Competing interests}

The authors declare that they have no competing interests.

\section{Authors' contributions}

KDD co-wrote the manuscript and performed the bioinformatic analysis. TS and wrote some of the analysis tools. KEH carried out the Bayesian analysis and co-wrote the manuscript. JAMF and CJL provided materials and assisted with study design. ME, FP, DY, GP provided materials and co-wrote the manuscript. TPS conceived the study, analysed the data and co-wrote the manuscript. All authors read and approved the final manuscript.

\section{Acknowledgements}

This research was supported in part by the National Health and Medical Research Council of Australia (628930 \& 65195), the Stop Buruli Initiative of the UBS-Optimus Foundation and the Victorian Government Department of Health. We are grateful to Scott Coutts and Jessica Porter for sequencing support, and Matthew Wakefield and Helen Lindsay for their expert assistance regarding PyCogent.

\section{Author details}

${ }^{1}$ Department of Microbiology and Immunology, University of Melbourne, Parkville, Australia. ${ }^{2}$ Victorian Bioinformatics Consortium, Monash University, Clayton, Australia. ${ }^{3}$ Victorian Infectious Diseases Reference Laboratory, North Melbourne, Australia. ${ }^{4}$ Department of Biomedical Sciences, Institute of Tropical Medicine, Antwerp, Belgium. ${ }^{5}$ Noguchi Memorial Institute for Medical Research, University of Ghana, Legon, Ghana. ${ }^{6}$ Swiss Tropical and Public Health Institute, Molecular Immunology, Basel, Switzerland. ' University of Basel, Basel, Switzerland.

Received: 25 March 2012 Accepted: 30 May 2012

Published: 19 June 2012

\section{References}

1. Stinear TP, Seemann T, Pidot S, Frigui W, Reysset G, Garnier T, Meurice G, Simon D, Bouchier C, Ma L, et al: Reductive evolution and niche adaptation inferred from the genome of Mycobacterium ulcerans, the causative agent of Buruli ulcer. Genome Res 2007, 17(2):192-200.

2. Stinear TP, Seemann T, Harrison PF, Jenkin GA, Davies JK, Johnson PDR, Abdellah Z, Arrowsmith C, Chillingworth T, Churcher C, et al: Insights from the complete genome sequence of Mycobacterium marinum on the evolution of Mycobacterium tuberculosis. Genome Res 2008, 18(5):729-741.

3. Sarfo FS, Le Chevalier F, Aka N, Phillips RO, Amoako Y, Boneca IG, Lenormand P, Dosso M, Wansbrough-Jones M, Veyron-Churlet $R$, et al: Mycolactone diffuses into the peripheral blood of Buruli ulcer patients-implications for diagnosis and disease monitoring. PLoS Negl Trop Dis 2011, 5(7):e1237.

4. Hong H, Coutanceau E, Leclerc M, Caleechurn L, Leadlay PF, Demangel C: Mycolactone Diffuses from Mycobacterium ulcerans-Infected Tissues and Targets Mononuclear Cells in Peripheral Blood and Lymphoid Organs. PLoS Negl Trop Dis 2008, 2(10):e325.

5. Stinear TP, Mve-Obiang A, Small PL, Frigui W, Pryor MJ, Brosch R, Jenkin GA, Johnson PD, Davies JK, Lee RE, et al: Giant plasmid-encoded polyketide synthases produce the macrolide toxin of Mycobacterium ulcerans. Proc Natl Acad Sci U S A 2004, 101(5):1345-1349.

6. Stinear TP, Hong H, Frigui W, Pryor MJ, Brosch R, Garnier T, Leadlay PF, Cole ST: Common evolutionary origin for the unstable virulence plasmid pMUM found in geographically diverse strains of Mycobacterium ulcerans. J Bacteriol 2005, 187(5):1668-1676.

7. Parkhill J, Sebaihia M, Preston A, Murphy LD, Thomson N, Harris DE, Holden MT, Churcher CM, Bentley SD, Mungall KL, et al: Comparative analysis of the genome sequences of Bordetella pertussis, Bordetella parapertussis and Bordetella bronchiseptica. Nat Genet 2003, 35(1):32-40.

8. Holt KE, Parkhill J, Mazzoni CJ, Roumagnac P, Weill FX, Goodhead I, Rance R, Baker S, Maskell DJ, Wain J, et al: High-throughput sequencing provides insights into genome variation and evolution in Salmonella Typhi. Nat Genet 2008, 40(8):987-993.

9. Hiller NL, Janto B, Hogg JS, Boissy R, Yu S, Powell E, Keefe R, Ehrlich NE, Shen $\mathrm{K}$, Hayes J, et al: Comparative genomic analyses of seventeen Streptococcus pneumoniae strains: insights into the pneumococcal supragenome. J Bacteriol 2007, 189(22):8186-8195.

10. Andersson JO, Andersson SG: Genome degradation is an ongoing process in Rickettsia. Mol Biol Evol 1999, 16(9):1178-1191.

11. Cole ST, Eiglmeier K, Parkhill J, James KD, Thomson NR, Wheeler PR, Honore N, Garnier T, Churcher C, Harris D, et al: Massive gene decay in the leprosy bacillus. Nature 2001, 409(6823):1007-1011.

12. Thomson NR, Howard S, Wren BW, Holden MT, Crossman L, Challis GL, Churcher C, Mungall K, Brooks K, Chillingworth T, et al: The complete genome sequence and comparative genome analysis of the high pathogenicity Yersinia enterocolitica strain 8081. PLOS Genet 2006, 2(12):e206.

13. Demangel C, Stinear TP, Cole ST: Buruli ulcer: reductive evolution enhances pathogenicity of Mycobacterium ulcerans. Nat Rev Microbiol 2009, 7(1):50-60.

14. Fyfe JAM, Lavender CJ, Handasyde KA, Legione AR, O'Brien CR, Stinear TP, Pidot SJ, Seemann T, Benbow ME, Wallace JR, et al: A Major Role for Mammals in the Ecology of Mycobacterium ulcerans. PLOS Negl Trop Dis 2010, 4(8):e791.

15. Kazumi Y, Ohtomo K, Takahashi M, Mitarai S, Sugawara I, Izumi J, Andoh A, Hasegawa H: Mycobacterium shinshuense isolated from cutaneous ulcer lesion of right lower extremity in a 37-year-old woman. Kekkaku 2004, 79:437-441.

16. Mve-Obiang A, Lee RE, Portaels F, Small PL: Heterogeneity of mycolactones produced by clinical isolates of Mycobacterium ulcerans: implications for virulence. Infect Immun 2003, 71(2):774-783.

17. Rhodes MW, Kator H, McNabb A, Deshayes C, Reyrat JM, Brown-Elliott BA, Wallace R Jr, Trott KA, Parker JM, Lifland B, et al: Mycobacterium pseudoshottsii sp. nov., a slowly growing chromogenic species isolated from Chesapeake Bay striped bass (Morone saxatilis). Int J Syst Evol Microbiol 2005, 55(Pt 3):1139-1147.

18. Stragier $P$, Ablordey A, Bayonne L, Lugor $Y$, Sindani I, Suykerbuyk $P$, Wabinga $H$, Meyers W, Portaels F: Heterogeneity among Mycobacterium ulcerans isolates from Africa. Emerg Infect Dis 2006, 12:844-847.

19. Ucko M, Colorni A, Kvitt H, Diamant A, Zlotkin A, Knibb WR: Strain variation in Mycobacterium marinum fish isolates. Appl Environ Microbiol 2002, 68(11):5281-5287. 
20. Kaser M, Rondini S, Naegeli M, Stinear T, Portaels F, Certa U, Pluschke G: Evolution of two distinct phylogenetic lineages of the emerging human pathogen Mycobacterium ulcerans. BMC Evol Biol 2007 7:177

21. Yip MJ, Porter JL, Fyfe JA, Lavender CJ, Portaels F, Rhodes M, Kator H, Colorni A, Jenkin GA, Stinear T: Evolution of Mycobacterium ulcerans and other mycolactone-producing mycobacteria from a common Mycobacterium marinum progenitor. J Bacteriol 2007, 189(5):2021-2029.

22. Röltgen K, Stinear TP, Portaels F, Certa U, Pluschke G: The genome, evolution and diversity of Mycobacterium ulcerans. Evol 2012, 12(3):522-529.

23. Kaser M, Hauser J, Small P, Pluschke G: Large sequence polymorphisms unveil the phylogenetic relationship of environmental and pathogenic mycobacteria related to Mycobacterium ulcerans. Appl Environ Microbiol 2009, 75(17):5667-5675.

24. Pidot S, Asiedu K, Kaser M, Fyfe J, Stinear T: Mycobacterium ulcerans and Other Mycolactone-Producing Mycobacteria Should Be Considered a Single Species. PLoS Negl Trop Dis 2010, 4(7):e663.

25. Parkhill J, Wren BW: Bacterial epidemiology and biology - lessons from genome sequencing. Genome Biol 2011, 12(10):230.

26. Qi W, Kaser M, Roltgen K, Yeboah-Manu D, Pluschke G: Genomic diversity and evolution of Mycobacterium ulcerans revealed by next-generation sequencing. PLOS Pathog 2009, 5(9):e1000580.

27. Pearson T, Busch JD, Ravel J, Read TD, Rhoton SD, U'Ren JM, Simonson TS, Kachur SM, Leadem RR, Cardon ML, et al: Phylogenetic discovery bias in Bacillus anthracis using single-nucleotide polymorphisms from whole-genome sequencing. Proc Natl Acad Sci U S A 2004, 101(37):13536-13541.

28. Portaels F, Meyers WM, Ablordey A, Castro AG, Chemlal K, de Rijk P, Elsen P, Fissette $K$, Fraga AG, Lee $R$, et al: First Cultivation and Characterization of Mycobacterium ulcerans from the Environment. PLoS Negl Trop Dis 2008, 2(3):e178.

29. De Gentile PL, Mahaza C, Rolland F, Carbonnelle B, Verret JL, Chabasse D: Cutaneous ulcer from Mycobacterium ulcerans. Apropos of 1 case in French Guiana. Bull Soc Pathol Exot 1992, 85(3):212-214.

30. Roltgen K, Qi W, Ruf M-T, Mensah-Quainoo E, Pidot SJ, Seemann T, Stinear TP, Kaser M, Yeboah-Manu D, Pluschke G: Single Nucleotide Polymorphism Typing of Mycobacterium ulcerans Reveals Focal Transmission of Buruli Ulcer in a Highly Endemic Region of Ghana. PLoS Negl Trop Dis 2010, 4(7):e751.

31. Stragier $P$, Hermans $K$, Stinear T, Portaels F: First report of a mycolactone-producing Mycobacterium infection in fish agriculture in Belgium. FEMS Microbiol Lett 2008, 286(1):93-95.

32. Tsukamura M, Kaneda $\mathrm{K}$, Imaeda T, Mikoshiba $\mathrm{H}$ : A taxonomic study on a mycobacterium which caused a skin ulcer in a Japanese girl and resembled Mycobacterium ulcerans. Kekkaku 1989, 64(11):691-697.

33. Trott KA, Stacy BA, Lifland BD, Diggs HE, Harland RM, Khokha MK, Grammer TC, Parker JM: Characterization of a Mycobacterium ulcerans-like infection in a colony of African tropical clawed frogs (Xenopus tropicalis). Comp Med 2004, 54(3):309-317.

34. Stinear TP, Jenkin GA, Johnson PD, Davies JK: Comparative genetic analysis of Mycobacterium ulcerans and Mycobacterium marinum reveals evidence of recent divergence. J Bacteriol 2000, 182(22):6322-6330.

35. Stragier P, Ablordey A, Meyers W, Portaels F: Genotyping Mycobacterium ulcerans and Mycobacterium marinum by using mycobacterial interspersed repetitive units. J Bacterio/ 2005, 187:1639-1647.

36. Krzywinski M, Schein J, Birol I, Connors J, Gascoyne R, Horsman D, Jones SJ, Marra MA: Circos: an information aesthetic for comparative genomics. Genome Res 2009, 19(9):1639-1645.

37. Tettelin H, Riley D, Cattuto C, Medini D: Comparative genomics: the bacterial pan-genome. Curr Opin Microbiol 2008, 11(5):472-477.

38. Mira A, Martin-Cuadrado AB, D'Auria G, Rodriguez-Valera F: The bacterial pan-genome:a new paradigm in microbiology. Int Microbiol 2010, 13(2):45-57.

39. Goris J, Konstantinidis KT, Klappenbach JA, Coenye T, Vandamme P, Tiedje JM: DNA-DNA hybridization values and their relationship to whole-genome sequence similarities. Int J Syst Evol Microbiol 2007, 57(Pt 1):81-91.

40. Yang F, Yang J, Zhang $X$, Chen L, Jiang Y, Yan Y, Tang X, Wang J, Xiong Z, Dong J, et al: Genome dynamics and diversity of Shigella species, the etiologic agents of bacillary dysentery. Nucleic Acids Res 2005, 33(19):6445-6458.

41. Huson DH: SplitsTree: analyzing and visualizing evolutionary data. Bioinformatics 1998, 14(1):68-73.

42. Pidot SJ, Porter JL, Tobias NJ, Anderson J, Catmull D, Seemann T, Kidd S, Davies JK, Reynolds E, Dashper S, et al: Regulation of the $18 \mathrm{kDa}$ heat shock protein in Mycobacterium ulcerans: an alpha-crystallin orthologue that promotes biofilm formation. Mol Microbiol 2010,

78(5):1216-1231.

43. Wiedenbeck J, Cohan FM: Origins of bacterial diversity through horizontal genetic transfer and adaptation to new ecological niches. FEMS Microbiol Rev 2011, 35(5):957-976.

44. Liu Y, Harrison PM, Kunin V, Gerstein M: Comprehensive analysis of pseudogenes in prokaryotes: widespread gene decay and failure of putative horizontally transferred genes. Genome Biol 2004, 5(9):R64

45. Rezwan M, Grau T, Tschumi A, Sander P: Lipoprotein synthesis in mycobacteria. Microbiol 2007, 153(Pt 3):652-658.

46. Stoll H, Dengjel J, Nerz C, Gotz F: Staphylococcus aureus deficient in lipidation of prelipoproteins is attenuated in growth and immune activation. Infect Immun 2005, 73(4):2411-2423.

47. Sander P, Rezwan M, Walker B, Rampini SK, Kroppenstedt RM, Ehlers S, Keller C, Keeble JR, Hagemeier M, Colston MJ, et al: Lipoprotein processing is required for virulence of Mycobacterium tuberculosis. Mol Microbiol 2004, 52(6):1543-1552

48. Bakala N'goma JC, Schue M, Carriere F, Geerlof A, Canaan S: Evidence for the cytotoxic effects of Mycobacterium tuberculosis phospholipase $C$ towards macrophages. Biochim Biophys Act 2010, 1801(12):1305-1313.

49. Dubey AK, Baker CS, Suzuki K, Jones AD, Pandit P, Romeo T, Babitzke P. CsrA regulates translation of the Escherichia coli carbon starvation gene, cstA, by blocking ribosome access to the cstA transcript. J Bacteriol 2003, 185(15):4450-4460.

50. Achard ME, Tree JJ, Holden JA, Simpfendorfer KR, Wijburg OL, Strugnell RA, Schembri MA, Sweet MJ, Jennings MP, McEwan AG: The multi-copper-ion oxidase CueO of Salmonella enterica serovar Typhimurium is required for systemic virulence. Infect Immun 2010, 78(5):2312-2319.

51. Rocha EP, Smith JM, Hurst LD, Holden MT, Cooper JE, Smith NH, Feil EJ: Comparisons of $\mathrm{dN} / \mathrm{dS}$ are time dependent for closely related bacterial genomes. J Theor Biol 2006, 239(2):226-235.

52. Agarwal N, Woolwine SC, Tyagi S, Bishai WR: Characterization of the Mycobacterium tuberculosis sigma factor SigM by assessment of virulence and identification of SigM-dependent genes. Infect Immun 2007, 75(1):452-461.

53. Raman S, Puyang X, Cheng TY, Young DC, Moody DB, Husson RN: Mycobacterium tuberculosis SigM positively regulates Esx secreted protein and nonribosomal peptide synthetase genes and down regulates virulence-associated surface lipid synthesis. J Bacteriol 2006, 188(24):8460-8468

54. Gold B, Deng H, Bryk R, Vargas D, Eliezer D, Roberts J, Jiang X, Nathan C: Identification of a copper-binding metallothionein in pathogenic mycobacteria. Nat Chem Biol 2008, 4(10):609-616.

55. Comas I, Chakravartti J, Small PM, Galagan J, Niemann S, Kremer K, Ernst JD, Gagneux S: Human T cell epitopes of Mycobacterium tuberculosis are evolutionarily hyperconserved. Nat Genet 2010, 42(6):498-503.

56. Hong H, Demangel C, Pidot SJ, Leadlay PF, Stinear T: Mycolactones: immunosuppressive and cytotoxic polyketides produced by aquatic mycobacteria. Nat Prod Rep 2008, 25(3):447-454.

57. Pidot SJ, Hong H, Seemann T, Porter JL, Yip MJ, Men A, Johnson M, Wilson P, Davies JK, Leadlay PF, et al: Deciphering the genetic basis for polyketide variation among mycobacteria producing mycolactones. BMC Genomics 2008, 9:462.

58. Hashimoto M, Tawaratsumida K, Kariya H, Kiyohara A, Suda Y, Krikae F, Kirikae T, Gotz F: Not lipoteichoic acid but lipoproteins appear to be the dominant immunobiologically active compounds in Staphylococcus aureus. J Immunol 2006, 177(5):3162-3169.

59. Ford CB, Lin PL, Chase MR, Shah RR, lartchouk O, Galagan J, Mohaideen N, loerger TR, Sacchettini JC, Lipsitch M, et al: Use of whole genome sequencing to estimate the mutation rate of Mycobacterium tuberculosis during latent infection. Nat Genet 2011, 43(5):482-486. 
60. Huber CA, Ruf MT, Pluschke G, Kaser M: Independent loss of immunogenic proteins in Mycobacterium ulcerans suggests immune evasion. Clin Vaccine Immunol 2008, 15(4):598-606.

61. Guenin-Mace L, Carrette F, Asperti-Boursin F, Le Bon A, Caleechurn L, Di Bartolo V, Fontanet A, Bismuth G, Demangel C: Mycolactone impairs T cell homing by suppressing microRNA control of L-selectin expression. Proc Nat Acad Sci 2011, 108(31):12833-12838.

62. Marsollier $L$, Brodin $P$, Jackson M, Kordulokova J, Tafalmeyer $P$, Carbonelle $E_{\text {, }}$ Aubry J, Milon G, Legras P, Saint Andre JP: Impact of Mycobacterium ulcerans biofilm on transmissibility to ecological niches and Buruli ulcer pathogenesis. PLoS Pathogens 2007, 3(5):e62.

63. NCBI Sequence Read Archive. http://www.ncbi.n/m.nih.gov/sra.

64. David M, Dzamba M, Lister D, Ilie L, Brudno M: SHRiMP2: Sensitive yet Practical Short Read Mapping. Bioinformatics 2011, 27(7):1011-1012.

65. Harrison P, Seemann T: Nesoni. http://www.vicbioinformatics.com/nesoni. shtml.

66. Huson DH, Bryant D: Application of Phylogenetic Networks in Evolutionary Studies. Mol Biol Evol 2006, 23(2):254-267.

67. Harris SR, Feil EJ, Holden MT, Quail MA, Nickerson EK, Chantratita N, Gardete S, Tavares A, Day N, Lindsay JA, et al: Evolution of MRSA during hospital transmission and intercontinental spread. Science 2010, 327(5964):469-474

68. Zerbino D, Birney E: Velvet: algorithms for de novo short read assembly using de Bruijn graphs. Genome Res 2008, 18(5):821.

69. Gladman S: VelvetOptimiser. http://bioinformatics.net.au/software. velvetoptimiser.shtml.

70. Sampson SL: Mycobacterial PE/PPE Proteins at the Host-Pathogen Interface. Clin Dev Immunol 2011, 2011:11.

71. Li W, Godzik A: Cd-hit: a fast program for clustering and comparing large sets of protein or nucleotide sequences. Bioinformatics 2006, 22(13):1658-1659.

72. Knight R, Maxwell P, Birmingham A, Carnes J, Caporaso JG, Easton BC, Eaton M, Hamady M, Lindsay H, Liu Z, et al: PyCogent: a toolkit for making sense from sequence. Genome Biol 2007, 8(8):R171.

73. Yap VB, Lindsay $H$, Easteal S, Huttley G. Estimates of the effect of natural selection on protein-coding content. Mol Biol Evol 2010, 27(3):726-734.

Cite this article as: Doig et al:: On the origin of Mycobacterium ulcerans, the causative agent of Buruli ulcer. BMC Genomics 2012 13:258.

\section{Submit your next manuscript to BioMed Central and take full advantage of:}

- Convenient online submission

- Thorough peer review

- No space constraints or color figure charges

- Immediate publication on acceptance

- Inclusion in PubMed, CAS, Scopus and Google Scholar

- Research which is freely available for redistribution 